

\section{DISCLAIMER}

This document was prepared as an account of work sponsored by the United States Govermment. Neither the United States Government nor any agercy thereof, nor The Regents of the University of California, nor any of their employees, makes any warranty, express or implied, or assumes any legal liability or responsibility for the accuracy, completeness, or usefulness of any information, apparatus, product, or process disclosed, or represents that its use would not infringe privately owned rights. Reference herein to any specific commercial product, process, or service by its trade name, trademark, manufacturer, or otherwise, does not necessarily constitute or imply its endersement, recommendation, or favoring by the United States Govemment or any agency therest, or The Regents of the University of California. The view's and opinions of authors expressed herein do not necessarily state or reflect those of the United States Government or any agency thereof or The Regents of the University of California and shall not be used for advertising or product endorsement purposes.

This report has been reproduced directly from the best available copy.

Available to DOE and DOE Contractors from the Office of Scientific and Technical Information P.O. Box 62, Oak Ridge, TN 37831

Prices available from (615) 576-8401, FTS 626-8401

Available to the public from the National Technical Information Service

U.S. Department of Commerce

5285 Port Royal Road, Springfield, VA 22161

Lawrence Berkeley Laboratory is an equal opportunity employer. 


\title{
Update to the RHIC Letter of Intent for An Experiment on Particle and Jet Production at Midrapidity
}

\author{
The STAR Collaboration
}

\author{
K. Kadija, G. Paic and D. Vranic \\ Rudjer Boskovic Institute, 41001 Zagreb, Yugoslavia \\ F.P. Brady, J.E. Draper and J.L. Romero \\ University of California, Davis, California 95616, U.S.A.
}

J. B. Carroll, V. Ghazikhanian, E. Gulmez, G.J. Igo, S. Trentalange and C. Whitten, Jr.

University of California, Los Angeles, California 90024, U.S.A.

M. Cherney

Creighton University, Omaha, Nebraska 68178, U.S.A.

S. Margetis, R.E. Renfordt, D. Röhrich, R. Stock, H. Ströbele and S. Wenig

University of Frankfurt, D-6000 Frankfurt am Main 90, West Germany

T. Hallman and L. Madansky

The Johns Hopkins University, Baltimore, Maryland 21218, U.S.A.

B. Anderson, D. Keane, R. Madey and J. Watson

Kent State University, Kent, Ohio 44242, U.S.A.

F. Bieser, M.A. Bloomer, D. Cebra, W. Christie, E. Friedlander, D. Greiner, C. Gruhn, J.W. Harris, H.Huang, P. Jacobs, S. Kleinfelder, P. Lindstrom, H. Matis, C. McParland, C. Naudet, G. Odyniec, D. Olson, A.M. Poskanzer, G. Rai, H.-G. Ritter, I. Sakrejda, J. Schambach, L.S. Schroeder, P.A. Seidl,

T.J.M. Symons, S. Tonse, H. Wieman and W.K. Wilson Lawrence Berkeley Laboratory, Berkeley, California 94720, U.S.A.

D.D. Carmony, Y. Choi, A. Hirsch, E. Hjort, N. Porile, R.P. Scharenberg,

B. Srivastava and M.L. Tincknell

Purdue University, West Lafayette, Indiana 47907, U.S.A.

\author{
A. D. Chacon and K. L. Wolf \\ Texas A \& M University, College Station, Texas 77843, U.S.A. \\ W. Dominik and M. Gazdzicki \\ Warsaw University, Warsaw, Poland \\ T. Pawlak, W. Peryt and J. Pluta \\ Warsaw University of Technology, Warsaw, Poland \\ W.J. Braithwaite, J.G. Cramer, D. Prindle and T.A. Trainor \\ University of Washington, Seattle, Washington 98195, U.S.A. \\ A. Breskin, R. Chechik, Z. Fraenkel, A. Shor and I. Tserruya \\ Weizmann Institute of Science, Rehovot 76100, Israel
}

July 1991

This work was supported by the Director, Office of Energy Research, Division of Nuclear Physics of the Office of High Energy and Nuclear Physics of the U.S. Department of Energy under Contract DE-AC03-76SF00098 


\title{
Update to the RHIC Letter of Intent for An Experiment on Particle and Jet Production at Midrapidity
}

\section{The $\Upsilon_{\text {STAR Collaboration }}$}

\author{
K. Kadija, G. Paic and D. Vranic \\ Rudjer Boskovic Institute, 41001 Zagreb, Yugoslavia
}

F.P. Brady, J.E. Draper and J.L. Romero

University of California, Davis, California 95616, U.S.A.

J. B. Carroll, V. Ghazikhanian, E. Gulmez, G.J. Igo, S. Trentalange and C. Whitten, Jr.

University of California, Los Angeles, Caiifornia 90024, U.S.A.

\section{Cherney}

Creighton University, Omaha, Nebraska 68178, U.S.A.

S. Margetis, R.E. Renfordt, D. Röhrich, R. Stock, H. Ströbele and S. Wenig University of Frankfurt, D-6000 Frankfurt am Main 90, West Germany

\section{T. Hallman and L. Madansky}

The Johns Hopkins University, Baltimore, Maryland 21218, U.S.A.

B. Anderson, D. Keane, R. Madey and J. Watson

Kent State University, Kent, Ohio 44242, U.S.A.

F. Bieser, M.A. Bloomer, D. Cebra, W. Christie, E. Friedlander, D. Greiner,

C. Gruhn, J.W. Harris, H. Huang, P. Jacobs, S. Kleinfelder, P. Lindstrom,

H. Matis, C. McParland, C. Naudet, G. Odyniec, D. Olson, A.M. Poskanzer,

G. Rai, H.-G. Ritter, I. Sakrejda, J. Schambach, L.S. Schroeder,

P.A. Seidl, T.J.M. Symons, S. Tonse, H. Wieman and W.K. Wilson

Lawrence Berkeley Laboratory, Berkeley, California 94720, U.S.A.

D.D. Carmony, Y. Choi, A. Hirsch, E. Hjort, N. Porile, R.P. Scharenberg,

B. Srivastava and M.L. Tincknell

Purdue University, West Lafayette, Indiana 47907, U.S.A.

A. D. Chacon and K. L. Wolf

Texas A \& M University, College Station, Texas 77843, U.S.A.

W. Dominik and M. Gazdzicki

Warsaw University, Warsaw, Poland

T. Pawlak, W. Peryt and J. Pluta

Warsaw University of Technology, Warsaw, Poland

W.J. Braithwaite, J.G. Cramer, D. Prindle and T.A. Trainor University of Washington, Seattle, Washington 98195, U.S.A.

A. Breskin, R. Chechik, Z. Fraenkel, A. Shor and I. Tserruya Weizmann Institute of Science, Rehovot 76100, Israel 


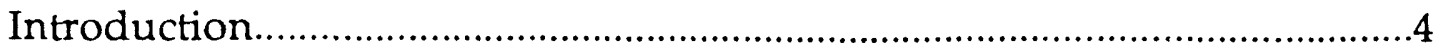

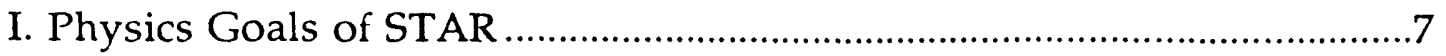

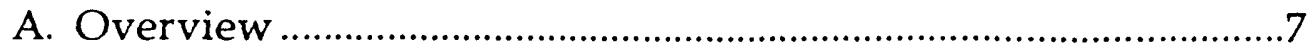

B. Strangeness and Charm Production ...............................................

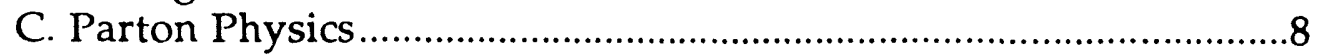

D. EM Energy ..................................................................................

E. Hanbury-Brown and Twiss (HBT) Interferometry ……...............10

II. Detector Systems and Physics Capabilities...................................................11

A. Time Projection Chamber (TPC) ...................................................11

TPC Performance.......................................................................11

Efficiencies of Track-Finding and Fitting .....................11

Primary Vertex Reconstruction....................................12

Overlapping Events ........................................................14

QED Electrons.................................................................15

TPC Operating Experience ………………………….................15

Space Charge ................................................................16

Comparison of Tracking - STAR, NA36, NA35.........16

Update on Electronics Costs (EOS/NA35/RHIC)...................17

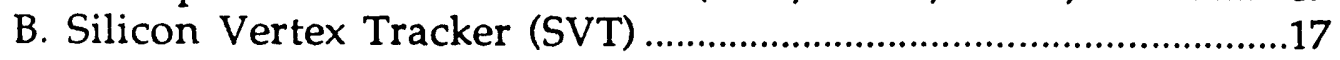

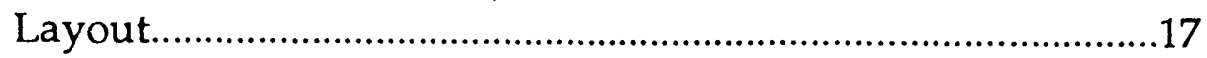

Primary and Secondary Vertex Capabilities...........................18

Track-matching Between the SVT and TPC.........................20

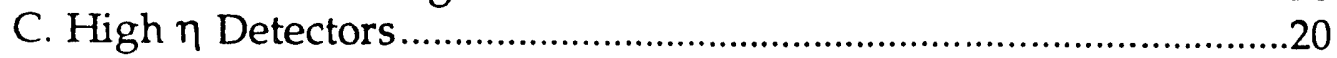

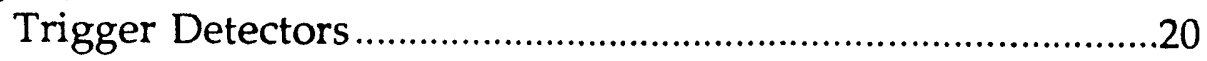

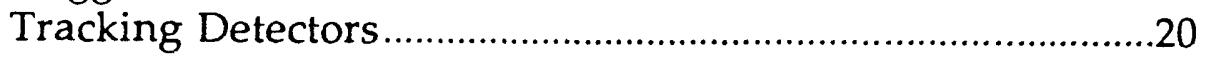

Proposed Layout.....................................................................

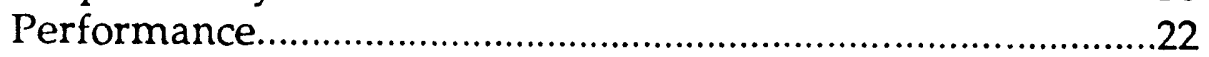

D. Time-of-Flight (TOF) Detectors …..............................................24

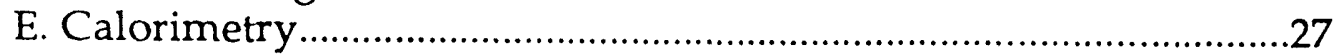

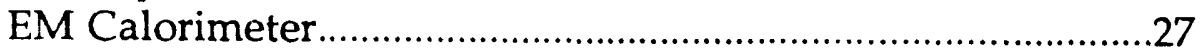

Hadron Calorimeter...............................................................22

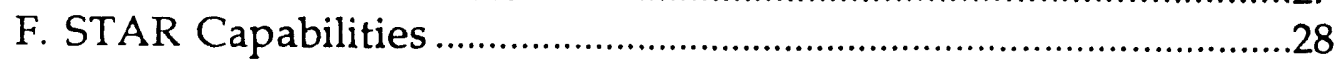

Acceptances for Tracking and PID........................................28

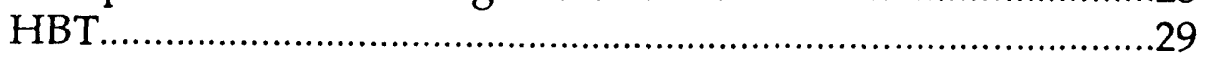

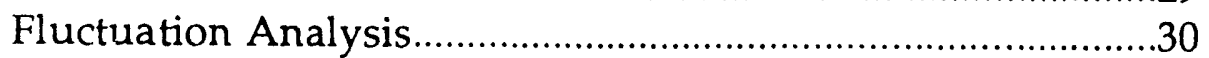

Long Range Fluctuations..............................................30

Electromagnetic/Hadronic Energy Balance ..................31

Short Range Fluctuations (Intermittency) ....................31

Jet Studies Using EM + Tracking...............................................31

Et Resolution Effects Due to Finite Acceptance ......................33

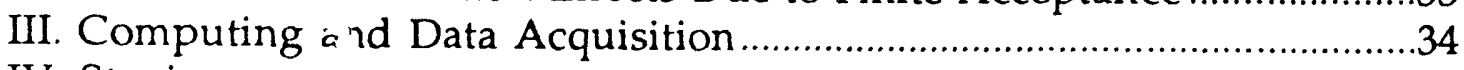

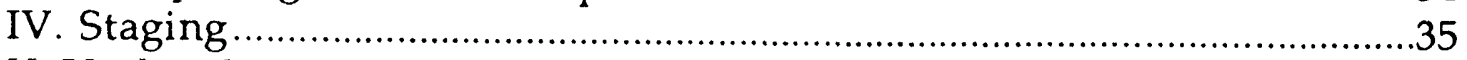

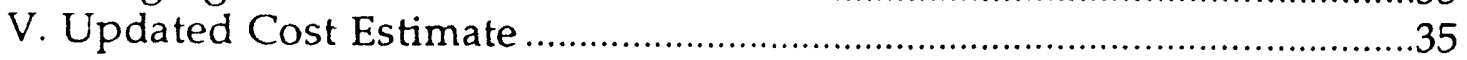

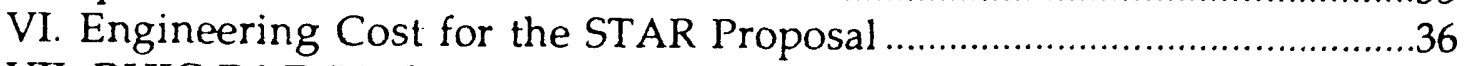

VII. RHIC R\&D Update .................................................................................

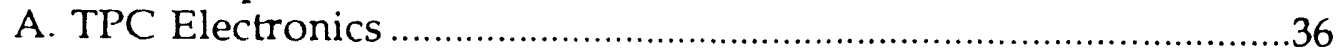




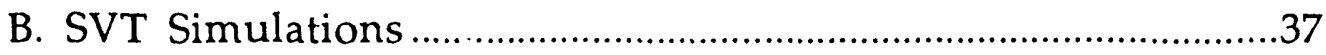

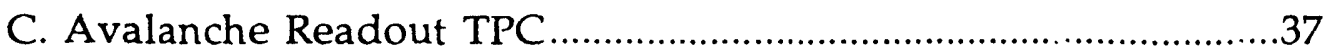

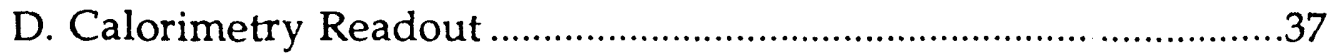

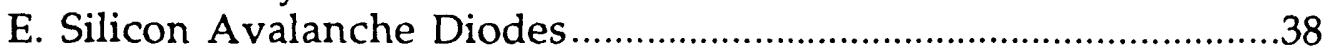

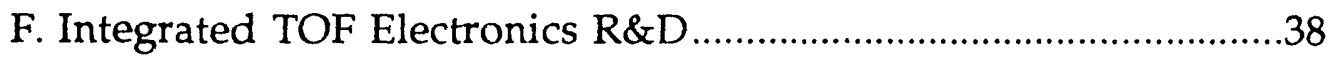

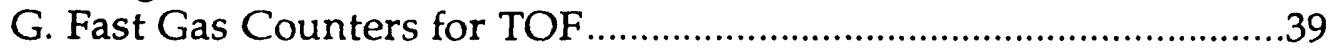

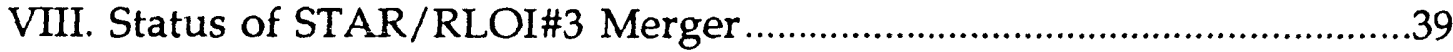

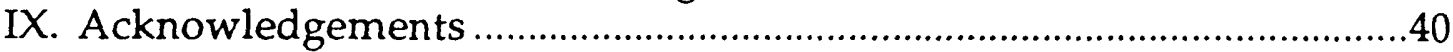

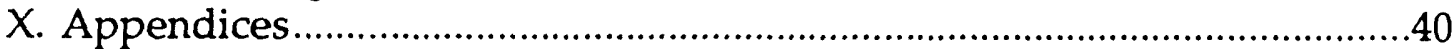

A. TPC Electronics Costs..............................................................40

B. Detailed Cost-Engineering Plan ................................................40 


\section{Introduction}

This document is an Update to the Letter of Intent (LOI) ${ }^{1}$ submitted by this collaboration in September 1990 proposing a large acceptance solenoidal tracking experiment. Since that time the collaboration has chosen the name STAR, representing Solenoidal Tracker $\underline{A} t \underline{R H I C}$. The physics interests of the STAR collaboration and the goals of the experiment are described in the original LOI. Since submission of the LOI, several changes have been made in the design of the experiment motivated by the desire to expand pseudorapidity coverage for complete event characterization and by the necessity to achieve cost reduction. The major changes arf. 1) expansion of pseudorapidity coverage to $|\eta|<4.5$ by the addition of tracking chambers external to the magnet; 2 ) reduction in the number of time-offlight channels from $100 \mathrm{~K}$ to $10 \mathrm{~K} ; 3$ ) staging implementation of hadronic calorimetry while using tracking and electromagnetic calorimetry to extract parton physics in the initial configuration; 4) reduction in the cost per channel of Time Projection Chamber (TPC) electronics through continued TPC electronics R\&D supported from RHIC funds; and 5) a change in the Silicon Vertex Tracker (SVT) technique to the use of silicon drift chambers. These changes and the status of ongoing R\&D programs to optimize the design and performance of the STAR experiment will be presented. In terms of detector performance it will be demonstrated that the space charge loading of the TPC is negligible and that the STAR experiment can operate at the highest RHIC luminosities. The configuration of the STAR experiment is shown in Fig. 1. A summary of the detector systems is presented in the table below.

${ }^{1}$ K. Kadija et al., RHIC Letter of Intent \#5, Lawrence Berkeley Laboratory Report, LBL-29651 (1990). 


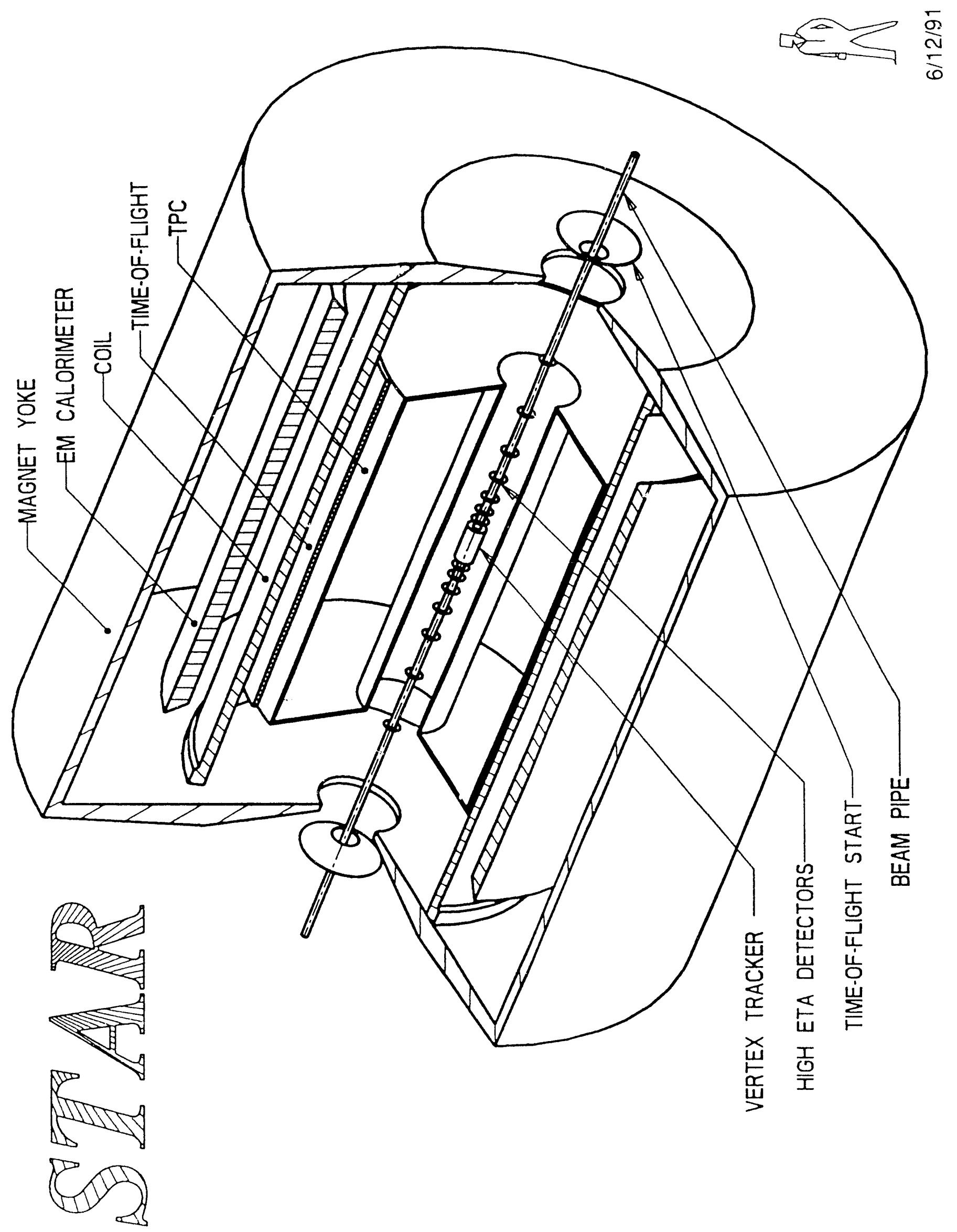

Fig. 1. The STAR experimental configuration. The external TPCS, which replace some of the high $\eta$ detectors, are not shown. 


\begin{tabular}{|c|c|}
\hline \multicolumn{2}{|c|}{ Overview of STAR Detector Systems } \\
\hline \multicolumn{2}{|l|}{ Tracking } \\
\hline \multicolumn{2}{|l|}{ Magnetic Field } \\
\hline Radius & $2.0 \mathrm{~m}$ \\
\hline Length & $8.0 \mathrm{~m}$ \\
\hline Strength & $0.5 \mathrm{~T}$ \\
\hline \multicolumn{2}{|l|}{ Time Projection Chamber (TPC) } \\
\hline Inner Radius & $0.5 \mathrm{~m}$ \\
\hline Outer Radius & $1.9 \mathrm{~m}$ \\
\hline Length & $5.0 \mathrm{~m}$ \\
\hline \# of Channels & 150,000 \\
\hline Acceptance & $|\eta| \leq 2.0$ \\
\hline \multicolumn{2}{|l|}{ High $\eta$ Detectors (8 TPC Sections) } \\
\hline Dimensions of Each $(\mathrm{L} \times \mathrm{W} \times \mathrm{D})$ & $1.8 \times 1.6 \times 1.0 \mathrm{~m}^{3}$ \\
\hline Distance of Front Face & $5.0 \mathrm{~m}$ \\
\hline Total \# of Channels & 7,200 \\
\hline Acceptance & $2.0 \leq|\eta| \leq 4.5$ \\
\hline \multicolumn{2}{|l|}{ Silicon Vertex Tracker } \\
\hline Type & Si Drift Chambers \\
\hline Radius 1st layer (double) & $5 \mathrm{~cm}$ \\
\hline Radius 2nd layer & $8 \mathrm{~cm}$ \\
\hline Radius 3rd layer & $11 \mathrm{~cm}$ \\
\hline Length & $40 \mathrm{~cm}$ \\
\hline \# of Channels & 36,000 \\
\hline Acceptance & $|\eta| \leq 2.0$ \\
\hline \multicolumn{2}{|l|}{ Time-of-Flight } \\
\hline Radius & $1.9 \mathrm{~m}$ \\
\hline \# of Channels & $1.0 \times 10^{4}$ \\
\hline Acceptance & $|\eta| \leq 1.0$ \\
\hline \multicolumn{2}{|l|}{ EM Calorimeter } \\
\hline Inner Radius & $2.2 \mathrm{~m}$ \\
\hline Thickness & $16.8 \mathrm{~cm}$ \\
\hline Depth Segmentation & 3-fold \\
\hline \# of Channels & $1.1 \times 10^{4}$ \\
\hline Acceptance & $\ln \mid \leq 1.05$ \\
\hline \multicolumn{2}{|l|}{ High $\eta$ Trigger } \\
\hline Type & Avalanche Chambers \\
\hline Segmentation & 10 in $\emptyset, 10$ in $\eta$ \\
\hline Acceptance & $1.5 \leq|\eta| \leq 4.0$ \\
\hline
\end{tabular}




\section{Physics Goals of STAK}

\section{A. Overvi $=w$}

The physics interests of the STAR collaboration have remained focussed on the original goals of our LOI. The STAR collaboration proposes a program to search for signatures of the Quark Gluon Plasma (QGP). The emphasis for this experiment is the correlation of many observables on an event-by-event basis. In the absence of definitive signatures for the QGP, it is imperative that such correlations be used to identify special events and possible signatures. Since the major detectors at RHIC will be expensive, a long-term physics program preferably of cross-disciplinary interest is important to their justification. The STAR program will necessarily require measurements of: 1) proton-proton, proton-nucleus and nucleus-nucleus interactions, 2) impact parameter dependence and 3) dependence on incident energy, in order to understand reactions at RHIC and thus detect and identify the QGP.

The large acceptance, good momentum and two-track resolution and particle identification capabilities of the STAR experiment facilitate the measurement of many observables, such as particle spectra, flavor composition, interferometry and density fluctuations of energy, entropy and multiplicity in azimuth and pseudorapidity. Measurements of the remnants of hard-scattered partons will be used as a penetrating probe of the QGP, and will provide important new information on the nucleon structure function and parton shadowing in nuclei.

The remainder of this section will be concerned with changes and evolution of the physics goals presented in the original LOI.

\section{B. Strangeness and Charm Production}

In addition to the measurement of the relative concentration of strange and nonstrange quarks ${ }^{2}$ on an event-by-event basis, as described in the original LOI, another unique feature of STAR is its ability to measure strange and anti-strange baryons (e.g., $\Lambda, \pi$ ) over a wide rapidity interval about midrapidity. Enhancements to the strange antibaryon content due to QGP formation have been predicted. ${ }^{3}$ Furthermore, multi-strange baryons $\left(\Xi^{-}, \Omega^{-}\right)$may be even more sensitive to the existence of the QGP. ${ }^{4}$ Measurements of strange and anti-strange baryons require detection of secondary decay vertices of particles with $\mathrm{c} \tau \sim 2-8 \mathrm{~cm}$. The combined tracking capabilities of the SVT and TPC detectors in the STAR experiment provide an azimuthally complete acceptance, which begins close to the interaction and extends to $>2 \mathrm{~m}$ distance, over a large rapidity interval. These qualities are essential for studying the production of strangeness.

\footnotetext{
2 B. Muller, "Hadronic Matter in Collisions 1988," World Scientific (1989), editors P. Carruthers and J. Rafelski, pg. 379 and references therein.

3 J. Rafelski and A. Schnabel, "Intersections Between Nuclear and Particle Physics," AIP Proceedings No. 176 (1988), G. M. Bunce (editor), pg. 1068, and references therein.

4 J. Rafelski, Phys. Rep. 88 (1982) 331.
} 
Measurement of "open charm" in collisions at RHIC 5 by detection of D-mesons could be extremely valuable in the search for the QGP. The STAR experiment, with its vertexing and multiparticle tracking capabilities, is the only system with the possibility of realizing this goal.

\section{Parton Physics}

RHIC will be the first accelerator to provide nuclear collisions at energies where perturbative QCD gives an accurate description of hard parton scattering, and where rates of detectable partonic debris (jets and high-pt particles) permit accurate measurements. If the structure functions of the colliding hadrons or nuclei are known, the rates of hard parton scattering can be calculated. This scattering occurs before hot matter forms in the region between the two receding nuclei. RHIC offers a unique opportunity to study the propagation of the partons travelling transversely in a highly excited medium and thereby to infer the properties of the medium. For example, it has been suggested that there will be observable changes in the energy loss of propagating partons as the energy density of the medium increases, particularly if the medium passes through a phase transition to the QGP. ${ }^{6}$ Energy loss in the medium results in jet quenching (i.e. a reduction of the jet yield at a given $p_{t}$ ) which has been observed in deep inelastic lepton scattering from nuclear targets. Jet quenching is expected to lead to significant effects in the spectra of single high $\mathrm{p}_{\mathrm{t}}$ particles, di-hadrons, and jets in AA collisions at RHIC. 6

Quark structure functions of hadrons and nuclei are being studied with deep inelastic lepton (DIS) scattering. However, the proton's gluon structure function and its change in nuclei (nuclear shadowing) can only be studied with a hadronic probe. Information on the gluon structure function of the proton at low Feynman $x$ is available from $\bar{p}$ p experiments. ${ }^{7}$ However, a complete picture, including the effect of nuclear shadowing, requires a systematic program of $\mathrm{pp}$ and $\mathrm{pA}$ studies at RHIC.

Reconstruction of the parton scattering kinematics is limited by acceptance and detector resolution effects, and by the superposition of particles from other incoherent processes, which occur during the collision. This latter problem is especially serious in high multiplicity events, where the jet can be entirely obscured. Therefore, the technique used to study parton dynamics depends on the colliding systems ( $p p, p A, A A$ ). The techniques of jet reconstruction and high $p_{t}$ particle measurements to extract parton information are being investigated with STAR.

The distribution of final state particles is represented by the fragmentation function. A few percent of jets fragment into a limited number of hard particles carrying most of the jet momentum. Observing only hard particles above some $\mathrm{p}_{\mathrm{t}}$ cut (e.g., $p_{t}>2 \mathrm{GeV}$ ) may solve the background problem for high multiplicity.

5 E.V. Shuryak, Phys. Lett. 78B (1978) 150; T. Matsui and H. Satz, Phys. Lett. 178B (1986) 416; A. Shor Phys. Lett. B215 (1988) 375 and Phys. Lett. B233 (1989) 231.

6 M. Gyulassy et al, Lawrence Berkeley Laboratory Report LBL-31002, to be published in Proc. of 4th Conference on the Intersections between Particle and Nuclear Physics, Tuscon, Arizona, 1991.

7 R. Baier and J.F. Uwens in "QCD Hard Partonic Processes", B. Cox ed., Plenum Press, New York and London (1987); J. Appel et al, Phys. Lett. B176, 239 (1982); E.L. Berger and J. Qiu, Proceedings of the Polarized Collider Workshop, AIP Proceedings 223, Collins et al editors (1991). 
However, in order to study parton dynamics in this way the fragmentation functions must be known. These functions are currently being studied by many groups and should be well known by the time RHIC experiments begin. These may change for fragmentation in the presence of a QGP or hadronic matter.

The measurement of structure functions for unpolarized $\mathrm{pp}$ collisions can be extended to the case of polarized beams. ${ }^{8}$ The importance of understanding how much of the proton spin is carried by the gluons has been emphasized by recent results in polarized DIS experiments, which can only study that portion carried by the quarks. ${ }^{9}$

The STAR experiment with its present complement of detectors will trigger on total $\mathrm{EM}_{\mathrm{t}}$ and high- $\mathrm{p}_{\mathrm{t}} \pi^{0} \mathrm{~s}$. Jet energies and single and multiple high- $\mathrm{p}_{\mathrm{t}}$ particles (including $\pi^{0} \mathrm{~s}$ ) will be measured. These capabilities apply for pp collisions and a range of $\mathrm{pA}$ and $\mathrm{AA}$ collisions. Achievable resolutions and the range of $\mathrm{A}$ that can be covered depend upon maximally efficient use of the EM calorimeter and tracking information.

Rates for Hard Processes

\begin{tabular}{|c|c|c|c|c|c|}
\hline Observable & $\begin{array}{c}\mathrm{NN}{\sqrt{s_{n n}}} \\
(\mathrm{GeV})\end{array}$ & System & $\mathscr{L}\left(\mathrm{cm}^{-2} \mathrm{~s}^{-1}\right)$ & $\begin{array}{l}p_{t} \text { Range } \\
(\mathrm{GeV} / \mathrm{c})\end{array}$ & $\# / 10^{7} \mathrm{~s}$ \\
\hline \multirow{9}{*}{$\begin{array}{c}\text { jets, } \\
\text { inclusive, } \\
|\eta|<0.5\end{array}$} & \multirow[t]{6}{*}{200} & \multirow[t]{2}{*}{$\mathrm{pp}$} & \multirow[t]{2}{*}{$5 \times 10^{30}$} & $>20$ & $2.3 \times 10^{6}$ \\
\hline & & & & $>40$ & $4.2 \times 10^{3}$ \\
\hline & & \multirow{2}{*}{$\begin{array}{c}\text { p Au } \\
\text { (Ref.10) } \\
\text { (min. bias) }\end{array}$} & \multirow[t]{2}{*}{$3.2 \times 10^{28}$} & $>20$ & $2.8 \times 10^{6}$ \\
\hline & & & & $>40$ & $5.0 \times 10^{3}$ \\
\hline & & \multirow{2}{*}{$\begin{array}{c}\text { Au Au } \\
\text { (central) }\end{array}$} & \multirow[t]{2}{*}{$2 \times 10^{26}$} & $>20$ & $5.5 \times 10^{5}$ \\
\hline & & & & $\geq 40$ & $1.0 \times 10^{3}$ \\
\hline & \multirow[t]{3}{*}{500} & \multirow[t]{3}{*}{$\mathrm{pp}$} & \multirow[t]{3}{*}{$1.4 \times 10^{31}$} & $>20$ & $1.1 \times 10^{8}$ \\
\hline & & & & $>40$ & $2.5 \times 10^{6}$ \\
\hline & & & & $>60$ & $1.3 \times 10^{5}$ \\
\hline \multirow[t]{9}{*}{$\pi^{0},|\eta|<1$} & \multirow[t]{6}{*}{200} & \multirow[t]{2}{*}{$\mathrm{pp}$} & \multirow[t]{2}{*}{$5 \times 10^{30}$} & $10 \pm .5$ & $4.8 \times 10^{4}$ \\
\hline & & & & $15 \pm .5$ & $1.5 \times 10^{3}$ \\
\hline & & \multirow{2}{*}{$\begin{array}{c}\mathrm{p} \mathrm{Au} \\
\text { (min. bias) }\end{array}$} & \multirow[t]{2}{*}{$3.2 \times 10^{28}$} & $10 \pm .5$ & $5.8 \times 10^{4}$ \\
\hline & & & & $15 \pm .5$ & $1.8 \times 10^{3}$ \\
\hline & & \multirow{2}{*}{$\begin{array}{c}\text { Au Au } \\
\text { (central) }\end{array}$} & \multirow[t]{2}{*}{$2 \times 10^{26}$} & $10 \pm .5$ & $1.2 \times 10^{4}$ \\
\hline & & & & $15 \pm .5$ & $\sim 350$ \\
\hline & \multirow[t]{3}{*}{500} & \multirow[t]{3}{*}{$\mathrm{pp}$} & \multirow[t]{3}{*}{$1.4 \times 10^{31}$} & $10 \pm .5$ & $4 \times 10^{6}$ \\
\hline & & & & $20 \pm .5$ & $3 \times 10^{4}$ \\
\hline & & & & $35 \pm .5$ & $\sim 1.2 \times 10^{3}$ \\
\hline
\end{tabular}

8 See LOI submitted by the RHIC Spin Collaboration.

9 European Muon Collaboration, J. Ásham et al, Phys. Lett. 206B (1988) 364 ; M.J. Alguard et al, Phys. Rev. Lett. 37 (1976) 1258; G. Baum et al. ibid. 51 (1983) 1153.

10 M.A. Bloomer, et al., in Fourth RHIC Workshop (1990), ENL 52262. Their Table 1 assumes a pp luminosity of $1.4 \times 10^{31} / \mathrm{cm}^{2} / \mathrm{sec}$ at $100 \mathrm{~A} \mathrm{GeV} / \mathrm{c}$; we have assumed a pp luminosity for this energy of $5 \mathrm{x}$ $10^{30} / \mathrm{cm}^{2} / \mathrm{sec}$. 
Some yields $/ 10^{7} \mathrm{sec}\left(\sim 100\right.$ days of RHIC running) for jets and single hard $\pi^{\circ} \mathrm{S}$ in the STAR detector at RHIC design luminosity are shown in the table above. These rates indicate that the programs outlined above can be carried out with significant staistics in reasonable time.

\section{EM Energy}

A unique feature of the STAR proposal is its emphasis on event-by-event correlations from data taken over the relatively large $\eta$ range of \pm 4.5 units. Correlation and fluctuation analyses in both azimuth and rapidity are improved considerably by combining the EM and charged particle data. Approximately $1 / 3$ of the energy is electromagnetic (EM) energy. The remaining hadronic energy can be measured by either calorimetry or charged particle tracking. In order to obtain reasonable energy resolution the fraction of EM energy must also be measured via calorimetry. More importantly, the measurement of EM energy vs charged-particle energy is one of the correlations which must be measured in the search for signatures of the QGP and other new physics. The unexplained imbalance between charged particle and neutral energy observed in Centauro and other cosmic ray events emphasizes the need for EM/charged particle measurements. ${ }^{11}$ Discussions of quark-gluon scattering within the QGP (eg., qg $\rightarrow \gamma q$ ) also point to the importance of measuring the electromagnetic energy as a possible signature of special events. ${ }^{12}$

\section{E. Hanbury-Brown and Twiss (HBT) Interferometry}

The STAR experiment, with its large acceptance and good momentum resolution at micirupidity, is especially well-suited for the investigation of collisions at RHIC using the techniques of Hanbury-Brown and Twiss (HBT) interferometry. The large number of midrapidity pions and kaons in central collisions of heavy nuclei may originate from a source which is large and/or long-lived, making HBT measurements difficult. Current theoretical predictions of the source size vary widely: the transverse radius $\left(R_{t}\right)$ of the pion source in central collisions of heavy nuclei could be as small as $10 \mathrm{fm}$ or as large as 30-40 fm.

The STAR detector will be able to measure transverse radii up to about $40 \mathrm{fm}$ on an ensemble basis for the bulk of the pion yield. Despite the enormous multiplicity of pions in a central event, it is still an open question whether an HBT analysis can be performed accurately on single events. However, the HBT analysis will certainly be possible for ensembles of events.

Kaon HBT interferometry is an important complement to pion interferometry since kaons are expected to be emitted significantly earlier, providing a picture of the time development of the source. In addition, resonance contributions to the pion and kaon correlation functions are substantially different, allowing a test of this imnortant correction. The STAR detector will have a significant capability for kaon

\footnotetext{
11 J.D. Bjorken and L.D. McLerran, Phys. Rev. D 20 (1979) 2353 and Y. Takahashi and S. Dake, Nucl. Phys. A461 (1987) 263C.

12 P.V. Rumskanen, IYFL-3-90): "Internátional Workshop on Quark-Gluon Plasma Signatures," Strasbourg, Frarice, Oct. 2-6 (1990).
} 
interferometry measurements: transverse radii of approximately $30 \mathrm{fm}$ can be measured at the peak of the kaon yield $\left(p_{t} \sim 500 \mathrm{MeV} / \mathrm{c}\right)$.

\section{Detector Systems and Physics Capabilities}

\section{A. Time Projection Chamber (TPC)}

\section{TPC Performance}

\section{Efficiencies of Track-Finding and Fitting}

The performance of the tracking algorithm in the STAR TPC was tested using $V_{s_{n n}}=200 \mathrm{GeV} \mathrm{Au}+\mathrm{Au}$ central events which were generated using the FRITIOF code and processed by the GEANT detector simulation program. Tracks were generated in the TPC and pad hits along with associated information from the tracks were passed to the reconstruction program.

The pattern recognition program for the STAR TPC is based on the ALEPH code, 13 modified for fixed target experiments by the BNL E810 group and tuned for high multiplicities in the CERN NA36 experiment. The track-finding program views the TPC in pseudo-rapidity slices as shown in Fig. 2.
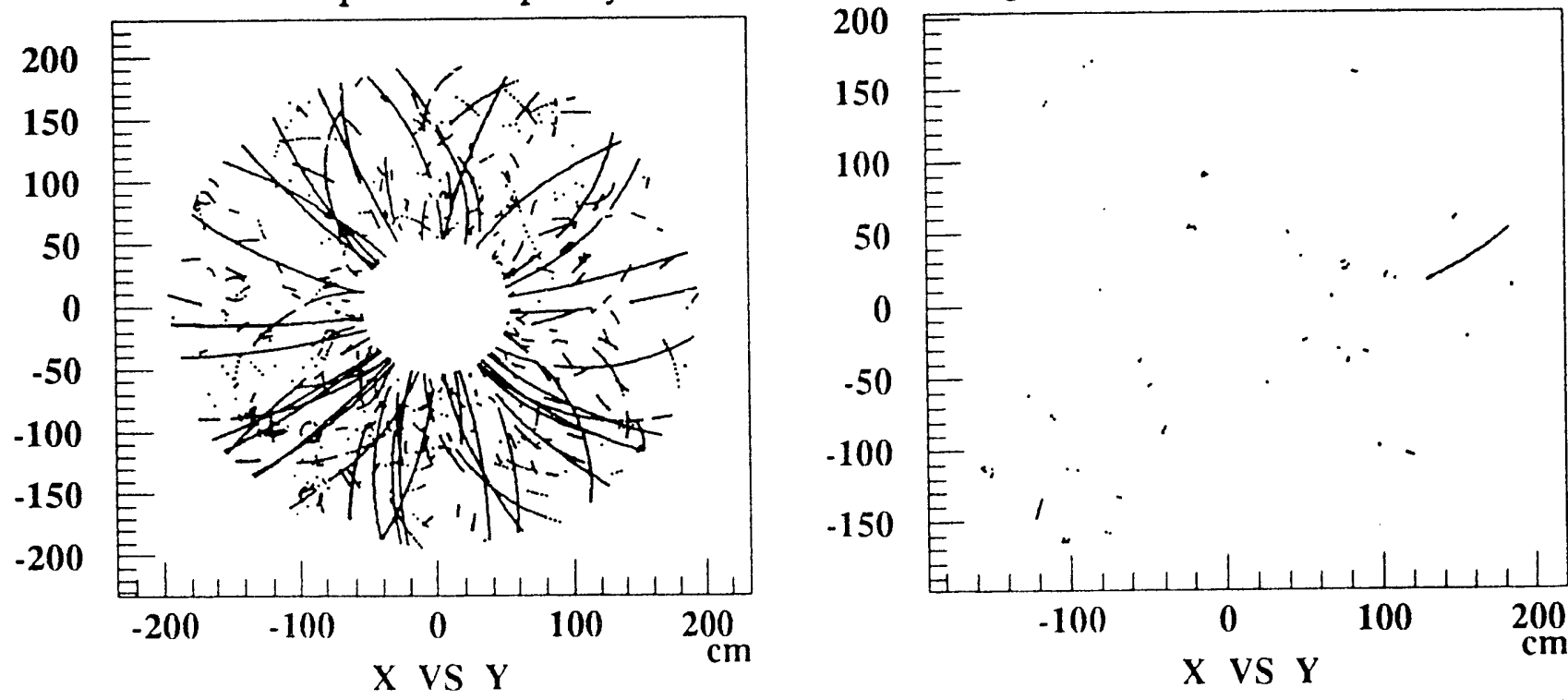

Fig. 2. Slice of 0.1 unit width about $\eta=1.0$ projected onto the TPC pad plane for a) reconstructed tracks, b) non-reconstructed tracks

Track formation starts at the outer radius of the TPC where the track density is lowest, i.e. $1 \%$ double occupancy in a $1^{\prime \prime} \times 1^{\prime \prime} \times 1^{\prime \prime}$ volume for central $\mathrm{Au}+\mathrm{Au}$ collisions. While stepping inward from one row to another toward the center of the chamber, the track following algorithm attempts to associate additional points with tracks. As the search proceeds inward track parameters are continuously updated, new tracks are added and candidate tracks which are too short are eliminated. By the

13 W.B. Atwood, et al.. Performance of the Aleph Time Projection Chamber - submitted to Nuclear Instruments and Methods $\mathrm{A}$. 
time the inner region of the TPC is reached, most of the tracks are well-defined. Therefore the higher hit density at the inner radius of the TPC (double occupancy up to $10 \%$ ) does not affect the performance of the algorithm for most tracks. Reconstructed tracks are fitted by a helix-fitting formula. ${ }^{14}$

On average the program successfully reconstructs the momentum of $90 \%$ of the generated tracks for central $\mathrm{Au}+\mathrm{Au}$ events. The $10 \%$ loss includes inefficiencies of the algorithm as well as low momentum tracks (mainly secondaries) with a radius that is too small to allow for reconstruction. Fig. 2 shows (a) reconstructed and (b) non-reconstructed tracks for a pseudorapidity slice. The quality of the results is shown in Fig. 3. An important criterion for determining the quality of the trackfinding and reconstruction is the ratio $R$ of good hits assigned to a reconstructed track to the total number of hits along the generated track. As can be seen from Fig. $3 b$ a majority of the tracks are found and reconstructed with only $1 \%$ of them having $\mathrm{R}<0.8$. The remaining background is caused by the above-mentioned tracks which are too short to reconstruct or which have very low momentum. The $\Delta p / p$ of the reconstructed tracks is found to be $1.1 \%$ in good agreement with an evaluation based on the track length and momentum distribution in an argon gas-filled TPC. 15 Tuning of the parameters of the tracking and reconstruction algorithms and incorporation of energy loss for the low momentum tracks can be expected to recover some of these tracks.
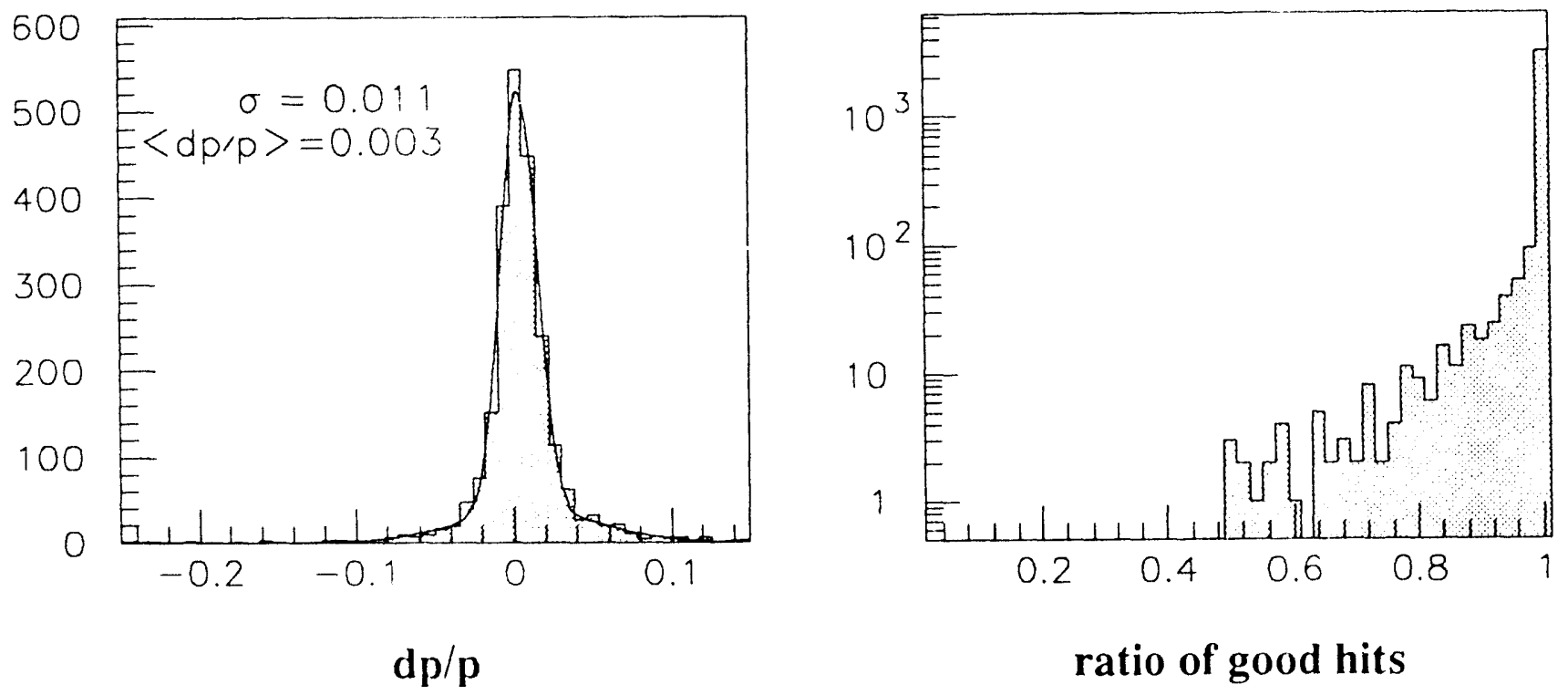

Fig. 3. Quality of reconstructed tracks for one event.

Primary Vertex Reconstruction

Reconstruction of the primary vertex is important for identifying multiple events in STAR. Tracks reconstructed in the TPC can be used to determine the

14 N.I. Chernov and G.A. Oskov, "Effective algorithms for circle fitting," Computer Physics Communications 33 (1984) 329-333.

15 R.K. Bock et al., Formula and Methods in Experimental Data Evaluation, European Physical Soriety, Geneva, CFRN (1984) 
primary vertex of the interaction. For the best vertex resolution only high momentum tracks, which suffer least from multiple scattering, should be used. Fig. 4 shows the accuracy of the primary vertex reconstruction when tracks with momentum in the bend plane $\mathrm{p}>800 \mathrm{MeV} / \mathrm{c}$ are used. When all tracks in an event are used, the corresponding widths $\sigma_{z}=0.1 \mathrm{~cm}$ and $\sigma_{x}=0.03 \mathrm{~cm}$ (as defined in Fig. 4) are found. These results will improve when more sophisticated methods of vertex finding ${ }^{16}$ are implemented.

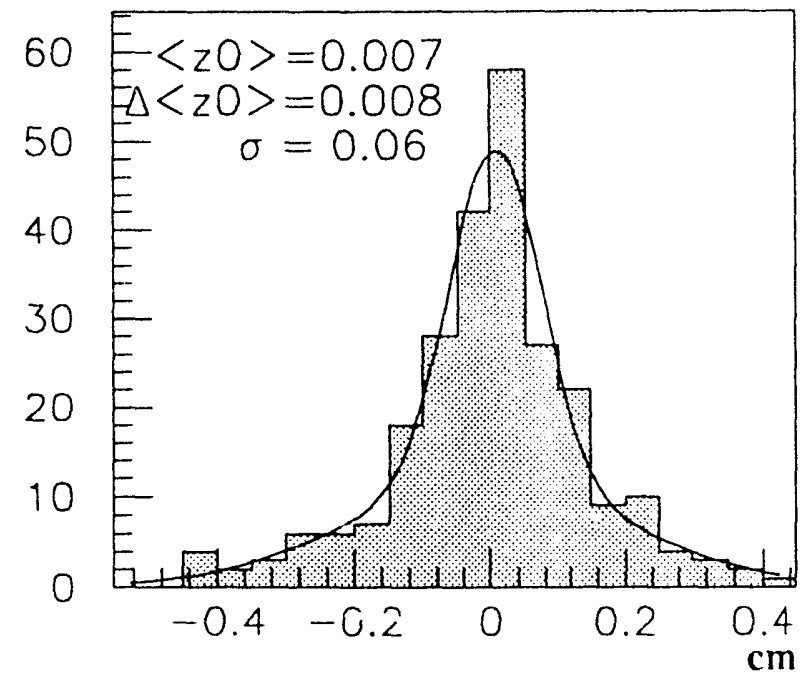

z0

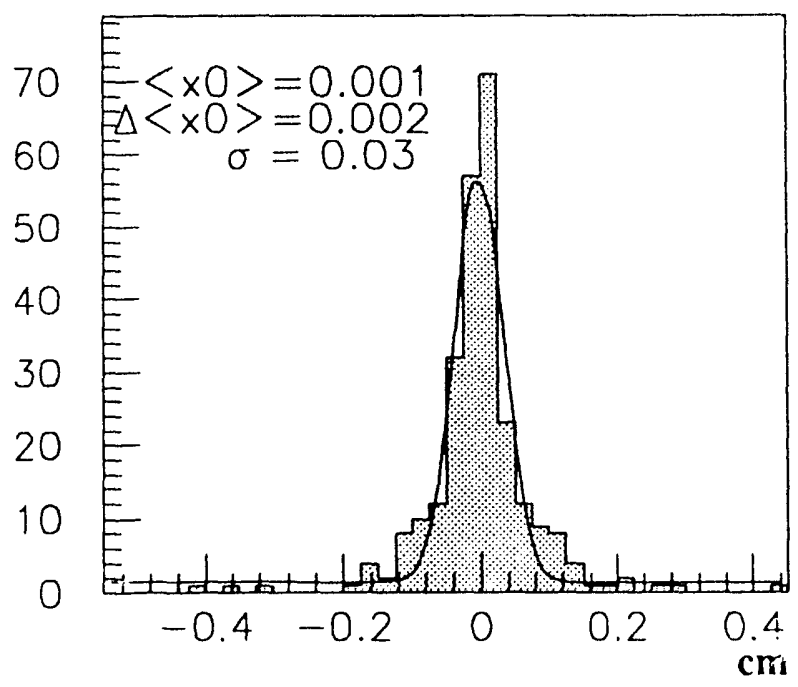

x0

Fig. 4. Primary vertex resolution in $z$ (along the beam direction) and $x$ with a momentum cut in the bend plane $>800 \mathrm{MeV} / \mathrm{c}$.

Due to the $50 \mu s$ readout time of the TPC, multiple events which occur within this time must be resolved. Results from studies of multiple events are shown in Fig. 5 for tracks with momentum in the bend plane p $>800 \mathrm{MeV} / \mathrm{c}$. From Fig. 5 it is seen that two events can be separated if they are more than $2 \mathrm{~mm}$ (FWHM $=2 \mathrm{~mm}$ for a single peak) apart. The corresponding widths of the vertices when all tracks are used in an event are slightly worse than in Fig. 5a with values of $\sigma=1.1-1.5 \mathrm{~mm}$. Furthermore, even if they are at the limit of this resolution (Fig. 5c), the structure of the two-event peak is distinctly different from that of a peak for a single event (Fig. 5b) of the same total multiplicity. It will be shown below that the vertex and overlapping event resolution is improved by adding the SVT information to that of the TPC.

\footnotetext{
16 E. Andersen et al., Determination of the Primary Vertex Position in the NA36 Experiment, Nucl. Instrum. and Methods Phys. Res. A, Accel. Spectrom. Detect. Assoc. Equip. A301 (1991) 69.
} 


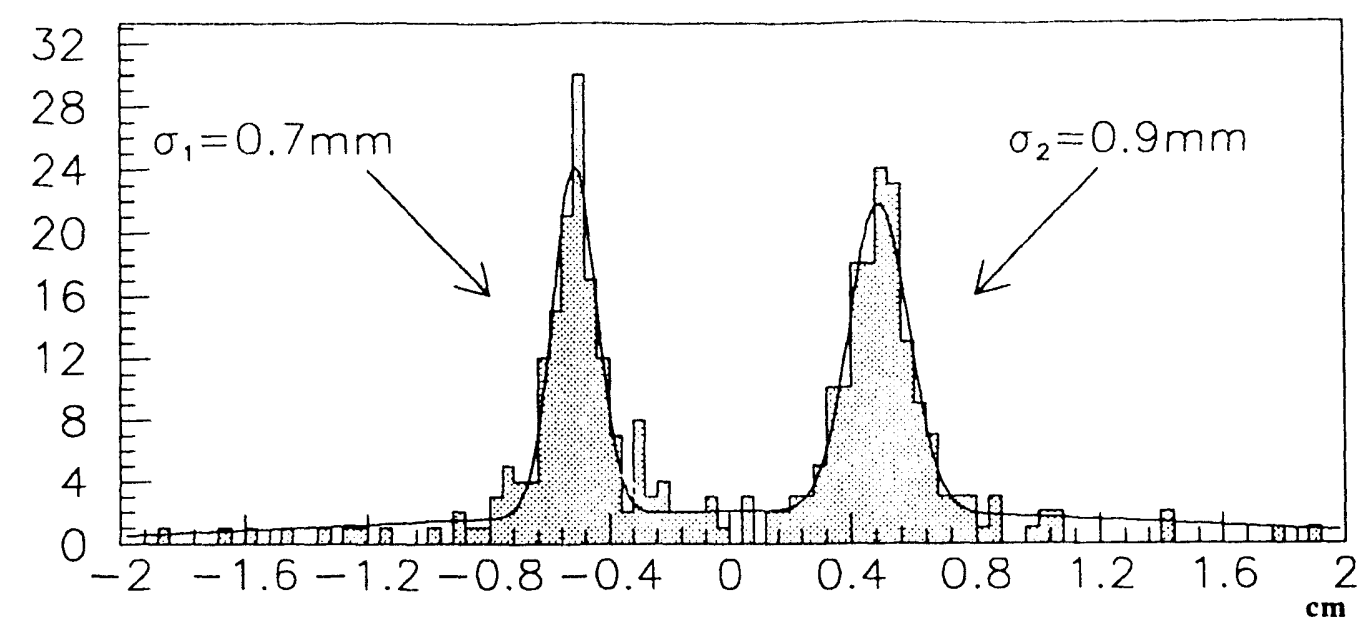

a) 2 events $1 \mathrm{~cm}$ apart

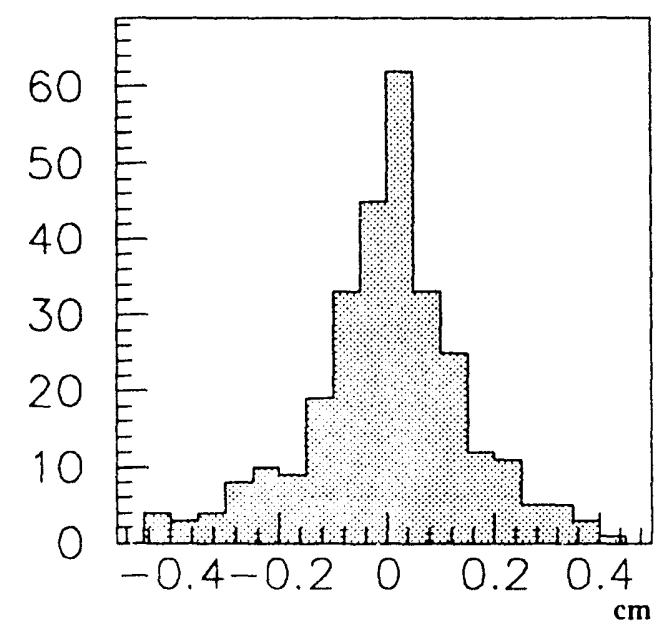

b) 1 event

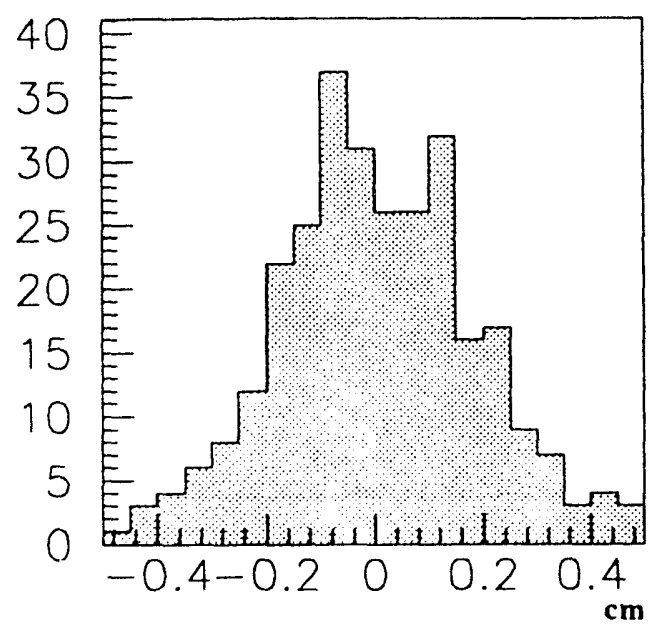

c) $2 \mathrm{ev} .2 \mathrm{~mm}$ apart

Fig. 5. Coordinate $z$ of the closest approach to the origin for tracks with a momentum in the bend plane greater than $800 \mathrm{MeV} / \mathrm{c}$ : a) for two events that overlap in the TPC with primary vertices $1 \mathrm{~cm}$ apart in $z$. b) for one event with the total multiplicity equal to the total multiplicity of the two overlapped events. c) for two events that overlap in the TPC with their primary vertices $2 \mathrm{~mm}$ apart in $z$.

\section{Overlapping Events}

Discrimination between two primary interactions originating from a single crossing of the circulating beams is dependent upon the vertex resolution and the timing characteristics of the tracking detectors. Discrimination and tracking of events overlapping in time during the $50 \mu \mathrm{sec}$ readout of the TPC was discussed above and in the LOI. At a luminosity an order of magnitude higher than the design luminosity for $\mathrm{Au}+\mathrm{Au}$ interactions at RHIC, events will start to overlap during the drift time of the TPC. However, only in the case when two central $\mathrm{Au}+\mathrm{Au}$ events occur in the same beam crossing at a distance closer than the detector capability to resolve primary vertices, does this become a problem. The probability of this is $1.6 \mathrm{x}$ $10^{-8}$ per central collision using the TPC vertex resolution and $1.6 \times 10^{-9}$ using that of the SVT. For pp collisions at design luminosity, the equivalent probability for two 
high multiplicity pp interactions $\left(5 \% \sigma_{R}\right)$ to occur in the same beam crossing with unresolvable individual vertices is $4 \times 10^{-6}$ using only the TPC and $4 \times 10^{-7}$ using the SVT.

\section{QED Electrons}

A concern which was not addressed in the original LOI was the potential background of low-mass lepton pairs ${ }^{17}$ produced by the electromagnetic interaction between the heavy ion colliding beams. Tables of the calculated double differential cross-sections $\left(d^{2} \sigma / d p_{t} d|y|\right)$ for $V_{s_{n n}}=200 \mathrm{GeV} A u+A u$ were obtained from $M$. Rhoades-Brown, integrated over the appropriate rapidity ranges for the TPC and SVT, and scaled by the design luminosity to calculate the flux of electrons expected in the detectors. Shown in Fig. 6 is the number of electrons per detector clearing time as a function of $p_{t}$ for the two devices.
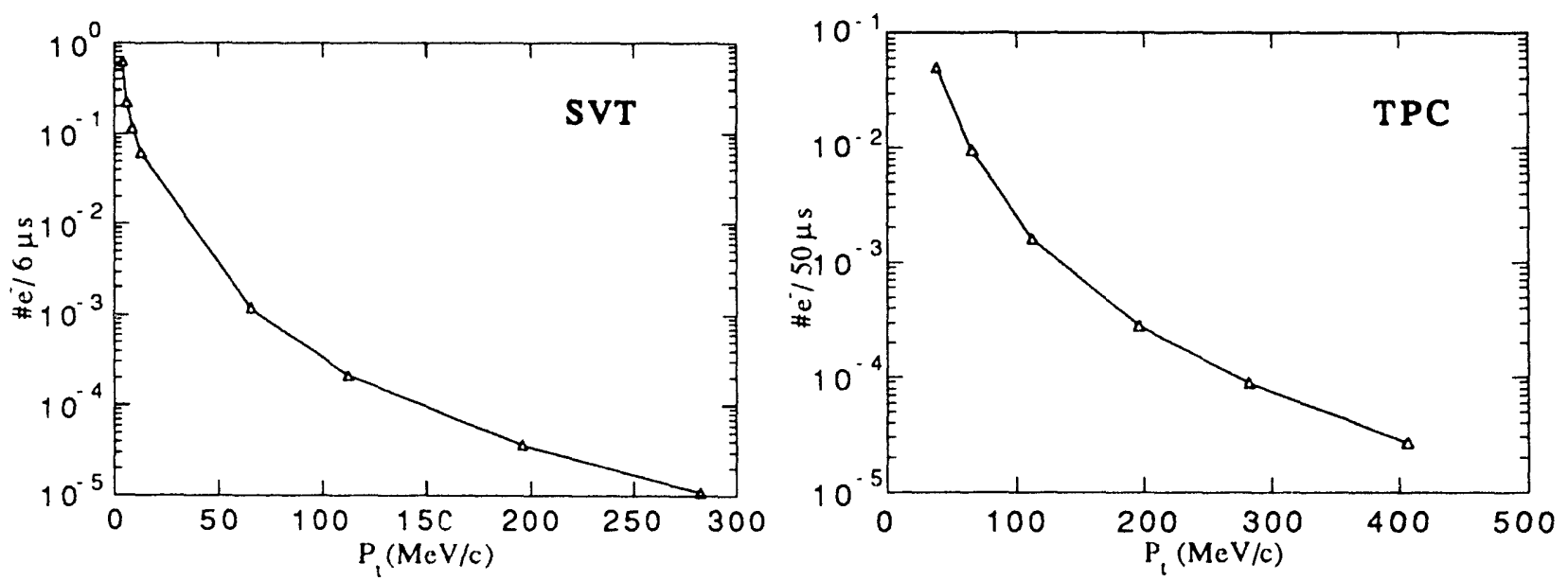

Fig. 6. Calculated number of electrons and positrons in the TPC and the SVT per detector clearing time.

It can be concluded that this source of background is not a problem for the proposed STAR configuration.

\section{TPC Operating Experience}

The STAR Collaboration has extensive experience in the design, construction and operation of TPCs in high energy heavy ion experiments. The CERN NA36 TPC, with its wire-readout, was the first TPC used in heavy ion experiments. The CERN NA35 TPC, installed in 1990, was the first TPC with pad readout used in heavy ion experiments. The EOS TPC, presently under construction at the Bevalac, is scheduled for operation at the end of 1991. Various members of the STAR Collaboration have played major roles in the development and operation of these TPCs for heavy ion experiments.

17 Mark J. Rhoades-Brown and T. Ludlam, Proceedings of Fourth Workshop on Experiments and Detectors for a Relativistic Heavy Ion Collider, July 2-7, 1990, p. 325. 


\section{Space Charge}

Since submission of the LOI, the effects of positive ion space charge in the TPC have been studied in detail. In the NA35 experiment at CERN, ${ }^{32} \mathrm{~S}$ beams at intensities of 25,000 ions/s were sent through the TPC to determine possible effects of distortions in the electric drift fields and the trajectories of tracks, due to positive ion space charge. This corresponds to $6 \times 10^{6}$ minimum ionizing particles per second in a cross sectional area of less than $1 \mathrm{~cm}^{2}$ in the TPC. No measurable distortions in the trajectories of tracks were observed.

The NA35 and EOS TPCs each use gated grids to reduce significantly positive ion reedback from the avalanche region near the sense wires. Tests have been performed on the new EOS gated grid and the results show that the suppression is much better than $10^{-4}$.

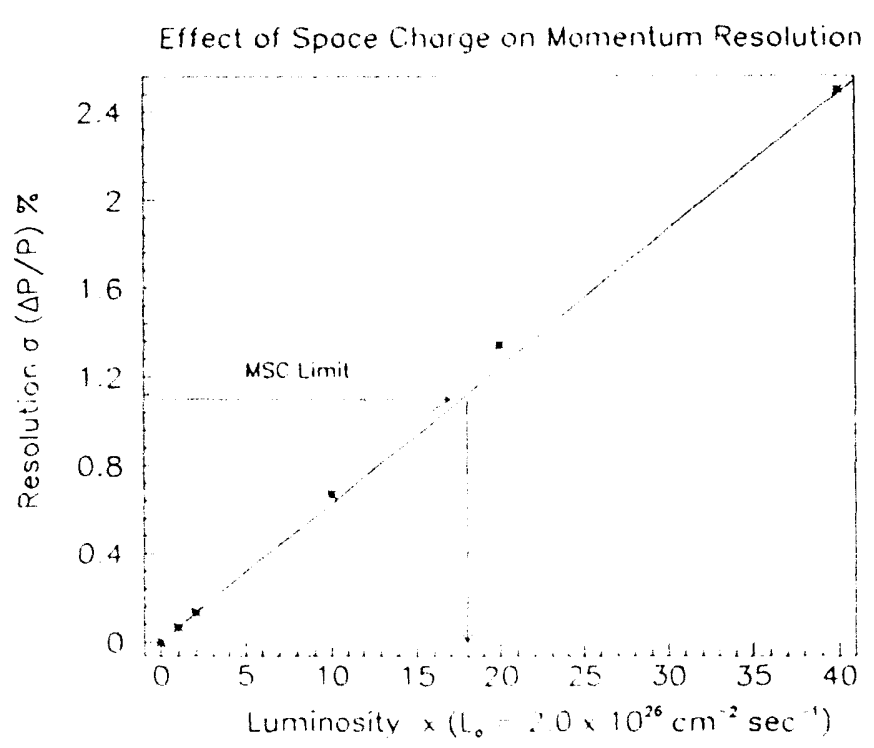

Fig. 7. The average momentum resolution due to space charge effects, as a function of luminosity in units of the design luminosity. The multiple scattering limit in argon gas is shown.

Furthermore, detailed r.umerical electrostatic calculations have been performed 18 to determine the effect of the primary ionization of the expected large multiplicity of charged particles in central collisions at RHIC. The accumulation of positive ion charge in the STAR TPC is found to have a small effect on the TPC spaci points for $\mathrm{Au}$ beam luminosities up to 20 times the design luminosity $\left(\mathscr{L}_{\mathrm{o}}=2 \mathrm{x}\right.$ $\left.10^{26} \mathrm{~cm}^{-2} \mathrm{sec}^{-1}\right)$. The electrostatic calculations show typical displacement errors at $\mathscr{L}_{\mathrm{o}}$ in the range $20-50 \mu \mathrm{m}$ depending on the track geometry. The rms resolution in $\Delta p / p$ due to space charge averaged over all reconstructed tracks in the TPC is found to be less than $0.07 \%$. Even at $10 \mathscr{L}_{\mathrm{o}}$ the performance of the TPC will not be degraded by this effect. Fig. 7 shows the averaged momentum resolution as a function of beam luminosity. The space charge distortions become comparable to multiple scattering at $\sim 18 \mathscr{L}_{0}$ for argon chamber gas and at $\sim 43 \mathscr{L}_{0}$ for neon.

\section{Comparison of Tracking - STAR, NA36, NA35}

Central collisions at RHIC are expected to produce several thousand particles per central collision. To see if the resulting track densities are manageable in the STAR TPC, they have been compared with those measured and successfully tracked in the CERN NA36 experiment. The STAR and NA36 TPCs have approximately the same track pair resolution for parallel tracks and both use analysis codes based on ALEPH tracking routines. In Fig. 8 the track densities are compared. For the NA36 data the

${ }^{18}$ K. Kadija et al., RHIC Letter of Intent \#5, Lawrence Berkeley Laboratory Report, LBL-29651 (1990). 


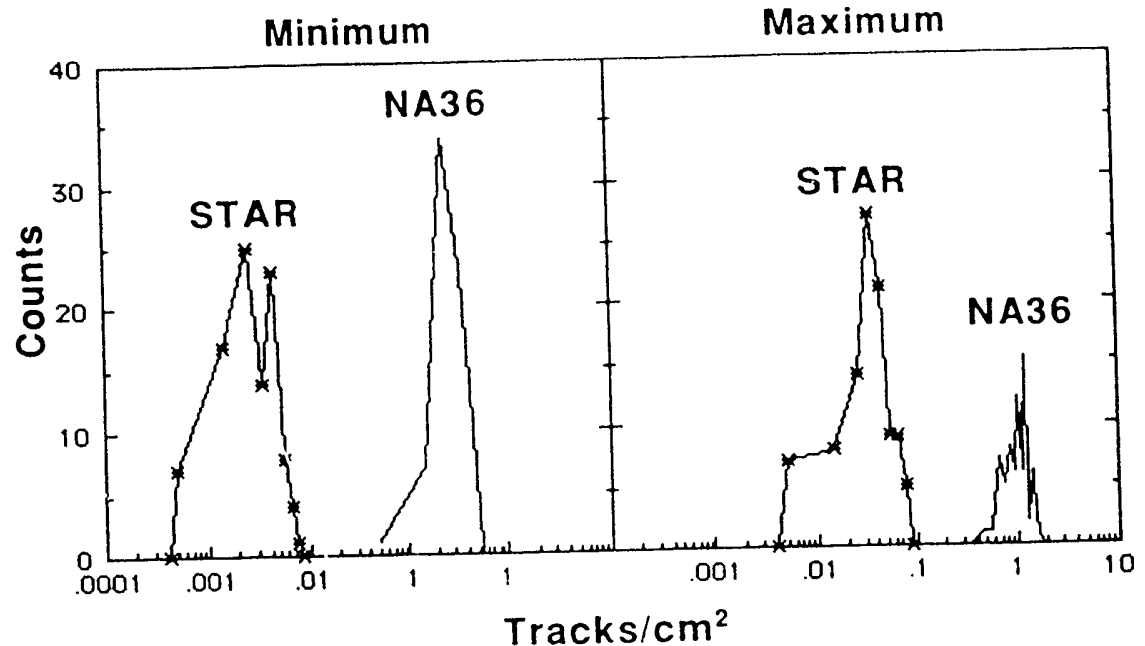

Fig. 8. Track densities observed in NA36 and expected in STAR in the TPC regions of minimum and maximum density. reconstructed track densities are plotted for central events. For comparison the track densities at the inner and outer radii of the STAR TPC are plotted for a central $\mathrm{Au}+\mathrm{Au}$ event. The track densities in STAR are expected to be up to two orders of magnitude lower in each region. The track densities measured successfully in the CERN NA35 TPC lie between the STAR and NA36 values.

Therefore, tracking in the STAR TPC at RHIC should present no problem even at track densities an order of magnitude higher than expected.

\section{Update on Electronics Costs (EOS/NA35/RHIC)}

The per channel cost of the TPC electronics has been reevaluated based on recent experience with EOS and NA35 TPC electronics. Using the EOS concept of performing amplification and digitization on the pad plane and using optical fiber links for the transport of the data off the detector, a cost of $\$ 30$ per channel is estimated for $150 \mathrm{~K}$ channels designed to handle 10 events/sec. A detailed cost breakdown is included in Appendix A. A large fraction of the cost is due to the need for extensive computing for compacting the data and centroid finding, whereas the fron! end cost can be kept low by the use of an integrated circuit for preamplification and shaping and a second one for analog storage and digitization.

\section{B. Silicon Vertex Tracker (SVT)}

\section{Layout}

A high spatial resolution device is necessary to achieve charged particle tracking at small distances from the primary vertex. This is particularly important for the measurement of strangeness and charm production. The STAR SVT has recently evolved from silicon strip detectors to silicon drift chambers. ${ }^{19,20,21}$ Silicon drift chambers are presently under development at BNL and have been incorporated recently into the NA45 experiment at CERN. Silicon drift chambers are an attractive option for a close-in tracking detector for the following reasons: 1) very high tracking precision ( 10 microns), 2) small dead time, 3 ) low number of electronic

19 P. Rehak et al., Nucl. Inst. and Meth. in Phys. Res. A248, (1986) 367.

20 E. Gatti, P. Rehak and M. Sampietro, Nucl. Inst. and Meth. in Phys. Res. A274, (1989) 469.

21 R. Rehak and E. Gatti, Nucl. Inst. and Meth. in Phys. Res. A289, (1990) 410. 


\begin{tabular}{|l|c|}
\hline \multicolumn{2}{|c|}{ Silicon Vertex Tracker } \\
\hline Inner radius & $5 \mathrm{~cm}$ \\
\hline Outer radius & $11 \mathrm{~cm}$ \\
\hline Length & $40 \mathrm{~cm}$ \\
\hline Total thickness & $900 \mu \mathrm{m}$ \\
\hline & $\sim 0.75 \% \mathrm{Lr}$ \\
\hline First layer of SDC Detector: & \\
\hline Number of sections & 6 \\
\hline Length of sections & $6.5 \mathrm{~cm}$ \\
\hline No. of anodes per section & 6,000 \\
\hline Position resolution & $\sim 10 \mu \mathrm{m}$ \\
\hline Pixel area & $0.0325 \mathrm{~mm}^{2}$ \\
\hline Cell occupancy & $0.1 \%$ \\
\hline
\end{tabular}

channels, 22 4) possibility of using modified TPC electronics to keep costs low, and 5) ability to monitor and calibrate ${ }^{23}$ the system. ${ }^{24}$

The SVT geometry takes full advantage of the relatively small size of the individual silicon drift chambers (SDC). The length of an individual silicon tile is $6.5 \mathrm{~cm}$ for silicon drift detectors made from 4inch diameter wafers. The thickness of a wafer is $\sim 300$ microns which appears to be a good compromise between signal strength (24K electrons are created by a minimum ionizing particle) and acceptable values of multiple scattering and secondary particle production for particles traversing the detector. Individual detectors are group $d$ into ladders $(6$ SDCs in each) and the ladders are arranged in three concentric barrels around the interaction region at radii of 5,8 and $11 \mathrm{~cm}$ (containing approximately $36 \mathrm{~K}$ channels of information). There will be a double layer at $5 \mathrm{~cm}$, which will allow readout in opposite directions to resolve overlapping events. Each barrel is about $40 \mathrm{~cm}$ in length, to nearly cover the diamond size. The SVT will be used in STAR to locate the main vertex precisely, identify secondary vertices and through track matching with the TPC, improve overall momentum resolution.

\section{Primary and Secondary Vertex Capabilities}

The FRITIOF event generator was used to generate $V_{s_{n n}}=200 \mathrm{GeV} \mathrm{Aul}+\mathrm{Au}$ central events. The events were then filtered through the STAR detector systems using GEANT. Multiple scattering, energy loss, secondary interactions and all other physics processes in GEANT were included. Fig. 9 demonstrates the capability of locating the primary vertex ( $\mathrm{z}$ is along the beam axis) with and without the SVT. Substantially improved resolution is obtained with the SVT. Tracks from events for which the primary vertices are separated by $2 \mathrm{~mm}$ can be associated with their

22 The position information is comparable to that of a silicon multistrip detector but the number of readout channels is reduced by nearly a factor of 1000 .

23 see Brookhaven National Laboratory Report BNL-45373.

24 This is important for understanding dependence of drift velocity on temperature and radiation damage. 


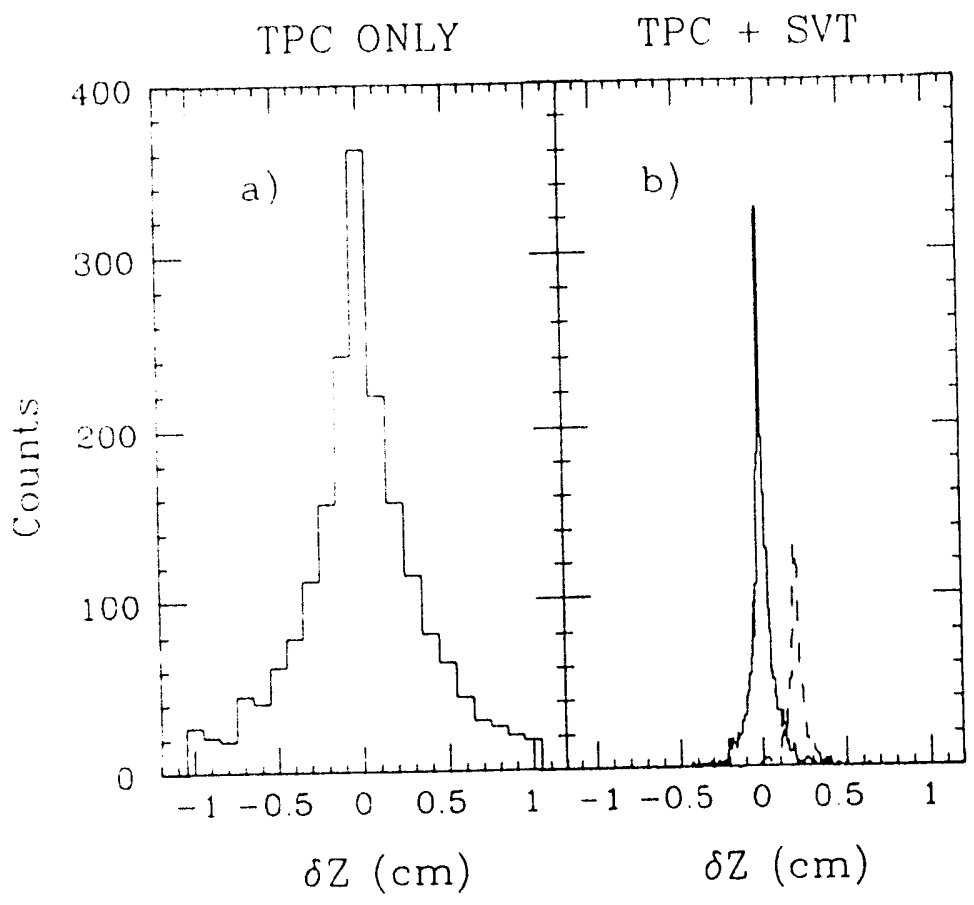

Fig. 9. Primary vertex resolution (along beam direction) using: a) TPC tracking only, b) TPC and SVT tracking.

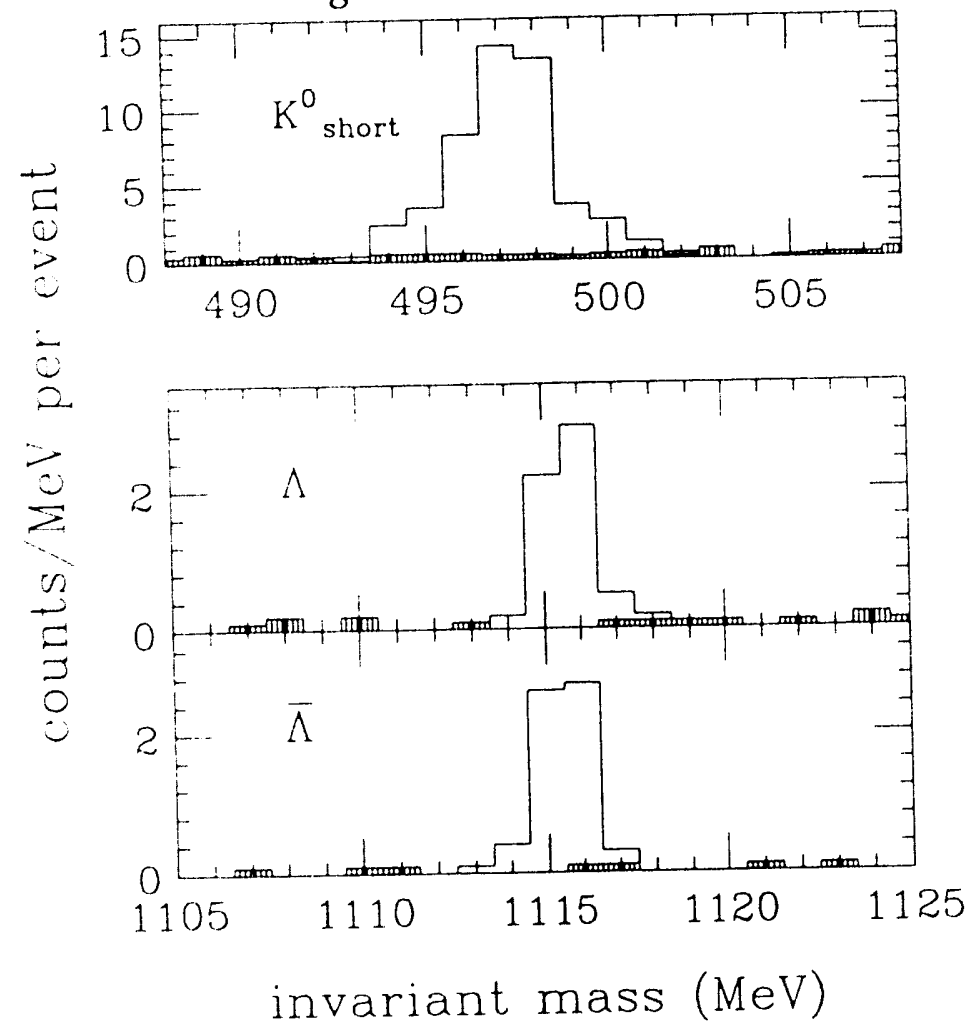

Fig. 10. Invariant mass plots reconstructed from full FRITIOF/GEANT events from central $\sqrt{s_{n n}}=$ $200 \mathrm{GeV} A u+A u$. Shaded boxes represent the contribution from random background. correct vertex as shown in Fig. 9b. Events with primary vertices as close as $200 \mu \mathrm{m}$ can be identified.

To study the ability of the SVT to measure secondary vertices ten FRITIOF events were used. $\mathrm{KO}_{s}, \Lambda$ and $\pi$ decays were studied. The spatial resolution of the SVT as well as interactions with material in STAR were taken into account. About $25 \%$ of $\mathrm{K}^{\circ} \mathrm{S}$ and $20 \%$ of $\Lambda$ and $\lambda$ are within the overall detector acceptance. The bulk $(>80 \%)$ of the losses are due to non-charged particle decays and the overall acceptance of STAR. To reduce the high density of potential crossing tracks near the primary vertex an additional cut of $1 \mathrm{~cm}$ for $\mathrm{Ko}_{\mathrm{s}}$ and $2 \mathrm{~cm}$ for $\Lambda$ and $\pi$ is applied (this is in effect a lifetime cut). In addition, the parent particle reconstructed from the two secondaries $\left(\mathrm{Ko}_{s} \rightarrow \pi^{+} \pi^{-}, \Lambda \rightarrow\right.$ $\mathrm{p} \pi^{-}$with $\Lambda \rightarrow \overline{\mathrm{p}} \pi^{+}$) is required to point back to the primary vertex within $2 \mathrm{~mm}$. Fig. 10 shows effective mass distributions (1 $\mathrm{MeV}$ bins) for the reconstructed events. Sharp peaks at the correct masses are obtained above a flat background. Approximately 2/3 of the accepted secondary decays are reconstructed. Presently we are working on: 1) charged hyperon decays (e.g., $\Xi^{-} \rightarrow \Lambda^{0} \pi^{-}$ with $\Lambda^{\circ} \rightarrow p \pi^{-}$), 2) the effects of PID resolution and track reconstruction efficiency in the evaluation of the SVT acceptance for these secondaries and 3) Dmeson decays. 


\section{Track-matching Between the SVT and TPC}

In order to utilize the combined power of the SVT and TPC, SVT hits must be assigned to tracks reconstructed in the TPC. Simulations have been carried out to address this task. The procedure involves the extrapolation of TPC helices to the SVT with the inclusion of multiple Coulomb scattering. It is found that with relatively simple algorithms there is a high efficiency for particles with $\mathrm{pt}_{\mathrm{t}}>250$ $\mathrm{MeV} / \mathrm{c}$ and that below $200 \mathrm{MeV} / \mathrm{c}$ the efficiency falls rapidly due to effects of multiple scattering. The overall SVT-TPC matching efficiency is $91 \%$ for a $p_{t} \geq 100$ $\mathrm{MeV} / \mathrm{c}$ and rises to $95 \%$ for $\mathrm{p}_{\mathrm{t}} \geq 200 \mathrm{MeV} / \mathrm{c}$.

\section{High $\eta$ Detectors}

\section{Trigger Detectors}

For triggering, the maximum possible $\eta$ region will be covered to facilitate comparisons between the results from nucleus-nucleus and proton-proton collisions. To cover the $\eta$ region from 1.5 to 4 , the use of avalanche gas multiplicity counters is proposed. The counters would be large disks operating just inside the iron yoke, but are not shown in any of the layouts. They would have 100 segments (10 in $\phi \times 10$ in $\eta)$ which provide fast (<100 ns) triggering signals and slower $(1 \mu \mathrm{sec})$ multiplicity information.

\section{Tracking Detectors}

A major evolution of the experimental configuration proposed for STAR is the addition of tracking detectors to cover the high $\eta$ regions (up to $|\eta|=4.5$ ). The maximum possible $\eta$-region will be covered to allow for extensive event characterization through complete multiplicity analysis, detailed study of the $\mathrm{dN} / \mathrm{d} \eta$ distribution, intermittency analysis, study of possible azimuthal asymmetries, and positive particle minus negative particle analysis for the study of the stopping of projectile nucleons. The high $\eta$ tracking chambers offer greatly increased coverage at a modest additional cost. The $\mathrm{dN} / \mathrm{d} \eta$ survey of the forward region facilitates the search for areas of enhanced particle production, possibly from QGP formation. Computer simulations to understand backgrounds produced by tracks from particle decays and interactions near the primary vertex are in progress.

\section{Proposed Layout}

The principal design under consideration for the high $\eta$ tracking detectors is a set of external TPCs. These devices would be placed in the region between the iron of the field return yoke and the first beamline dipole magnet $(B C 1)$ as shown in Fig. 11. In the first design the TPCs would consist of four modules, each $1.6 \mathrm{~m} \times 1.8$ $m$ rectangles and arranged to form a $3.4 \mathrm{~m} \times 3.4 \mathrm{~m}$ square with a $20 \mathrm{~cm} \times 20 \mathrm{~cm}$ square hole in the center for the beamline. They cover the $|\eta|$ range from 2.0 to 4.5 . Alternat.vely, external TPCs with a radial geometry are being considered. The attractive features of a design incorporating radial drift are the azimuthal symmetry and the reduced effective pixel size at smaller radii. This configuration would provide smaller pixels where the hit density is the highest and the highest position resolution where it is necessary, at high $\eta$ where the tracks experience the least 
deflection due to the magnetic field. However, there are technical details that must be addressed through prototyping before this technique can be implemented.

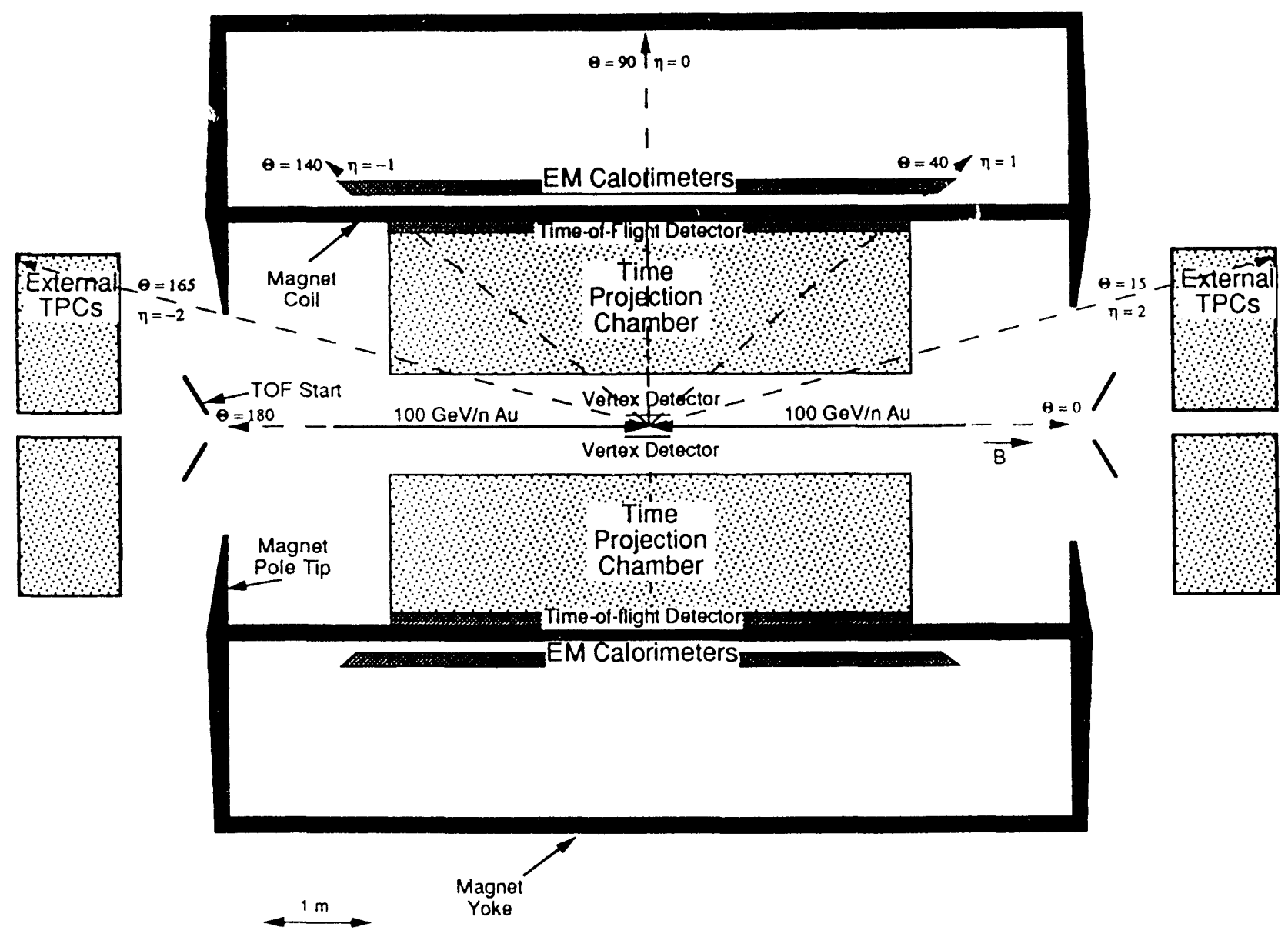

Fig. 11. A view of the experimental configuration showing the position of the external TPCs. 
The electronics for the external TPCs would be identical to those of the main TPC. Since the drift distance is shorter for the external TPCs, a slow cool gas mixture can be used. This will minimize diffusion which might otherwise have been a problem since there is no parallel magnetic field to help constrain the ionization cloud. The hit density at high $\eta$ will determine how close to the beam pipe the external TPCs can operate. To estimate the anticipated particle flux, events were generated using FRITIOF for $\sqrt{s_{n n}}=200 \mathrm{GeV} / \mathrm{n}$ Au $+\mathrm{Au}$ central collisions. These events were then passed through a simulation of the detector configuration using GEANT. We estimate that the hit density will be $0.5 \mathrm{tracks} / \mathrm{cm}^{2}$ at a radial distance of $4 \mathrm{~cm}$ from the beam axis. The ability to differentiate adjacent tracks will be determined by the diffusion of the ionization cloud, the partitioning of the signal across multiple anodes, and the effective 'pixel' size.

\section{Performance}

The FRITIOF+GEANT simulations predict that approximately half of the particle flux at high $\eta$ will be due to secondary tracks. These are the result of interactions with the beam pipe, the walls of the main TPC, the SVT, the iron field return yoke, and the decays of unstable particles. To accurately measure the distribution of particles from the reaction these secondary particles must be distinguished from the primaries. Therefore, tracking chambers must be used at high $\eta$ and not counters which simply record flux patterns. All primary particles will point back to the main vertex, while particles created in secondary interactions will point back to a random location along the beamline. Due to the solenoidal field of the STAR magnet, the primary particles can follow a complex trajectory before hitting the external TPCs. However, most primary particles at high $\eta$ are very stiff and will not deviate much from a direct line of flight. Therefore, vectoring can be used to reject the majority of secondary particles $(90 \%$ are rejected in the simulations). The requirement that the primary tracks are stiff places a cutoff at $p_{z}=$ $500 \mathrm{MeV} / \mathrm{c}$ and results in fewer than $2 \%$ of the primary tracks being rejected.

Vectoring can also be used to reject tracks from overlapping events. Any interaction that occurs at a time that is different from that for which the system was triggered will be reconstructed incorrectly in the drift direction and thus will not point back toward the main vertex. These tracks will be rejected in the same manner as secondary interaction tracks. This will allow for rejection of tracks that came from interactions that occurred within one $\mu$ sec of the event of interest. Although the TPC+SVT combination has better overlapping event rejection, the high $\eta$ tracking detectors are important for this purpose in minimum bias events and events where the number of tracks emitted at midrapidity is small. The $\mathrm{dN} / \mathrm{d} \eta$ distribution of primary tracks incident on the main and external TPCs and the distribution reconstructed after tracking are displayed in Fig. 12. The reconstructed distribution includes the loss of primary tracks due to the low $\mathrm{p}_{z}$ cutoff and due to the two-track resolution. Also included is the false addition of tracks associated with decays of unstable particles. These come mostly from $\mathrm{K}_{\mathrm{s}} \rightarrow \pi^{+} \pi^{-}$decays. 
Ouler Histogrom is Charged input from FRITIOF

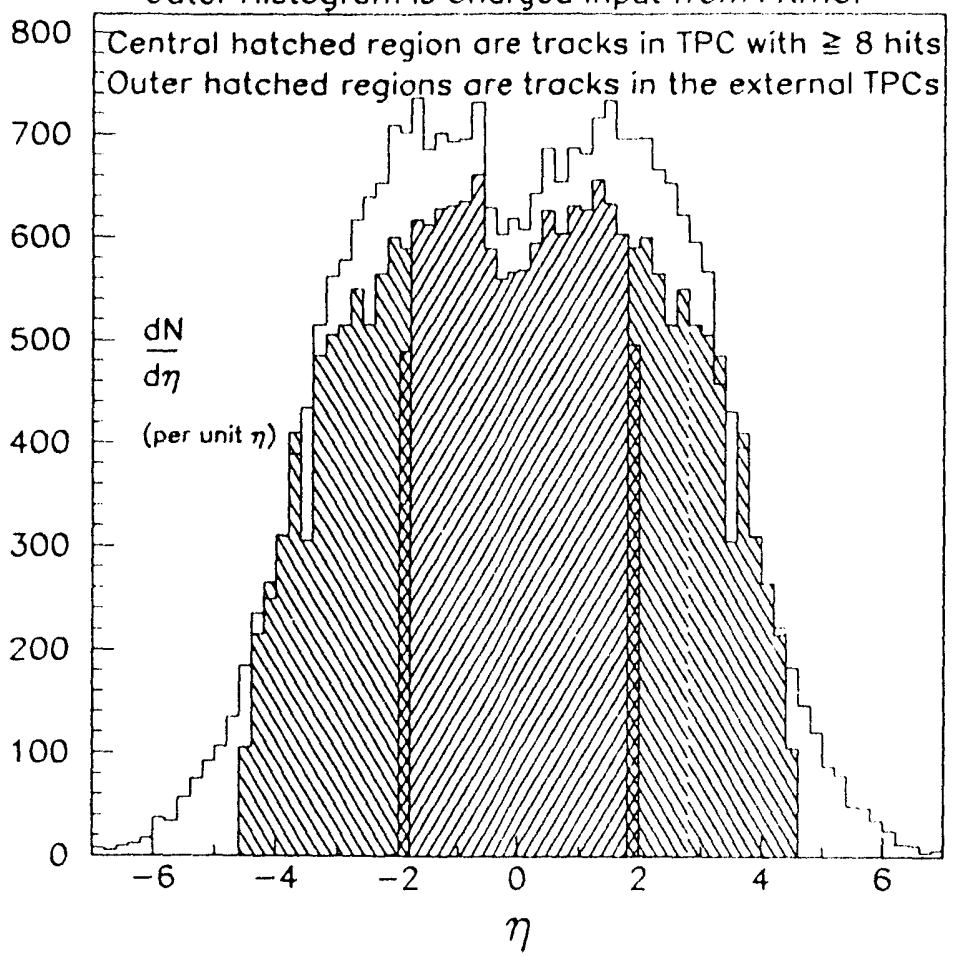

Fig. 12. The outer histogram displays the $d N / d \eta$ distribution that is input from the FRITIOF event generator. The hatched histograms represent the $d N / d \eta$ distributions reconstructed from tracking in the central and external TPCs.

The sign of the charge of the primary tracks can be identified over a range of momenta. This range of momenta is limited by the uncertainty due to multiple Coulomb scattering within the intervening material and by the measurement precision of the external TPCs. Fig. 13 indicates the momenta which can be charge identified for a given $\eta$. Fig. 14 indicates the distribution of excess positive charge, positive - negative, that could be observed in the case of a radial TPC with $|\eta|<5.5$.

Fig. 13. The region for which the external TPCs can identify the charge of incident tracks is bounded in $\eta$ by the physical limits of the devices and in momentum by the effect of the magnetic field. For low $p_{z}$ tracks, the particles execute multiple orbits and therefore cannot be identified. High $p_{z}$ tracks experience too little bending for charge to be resolved. 


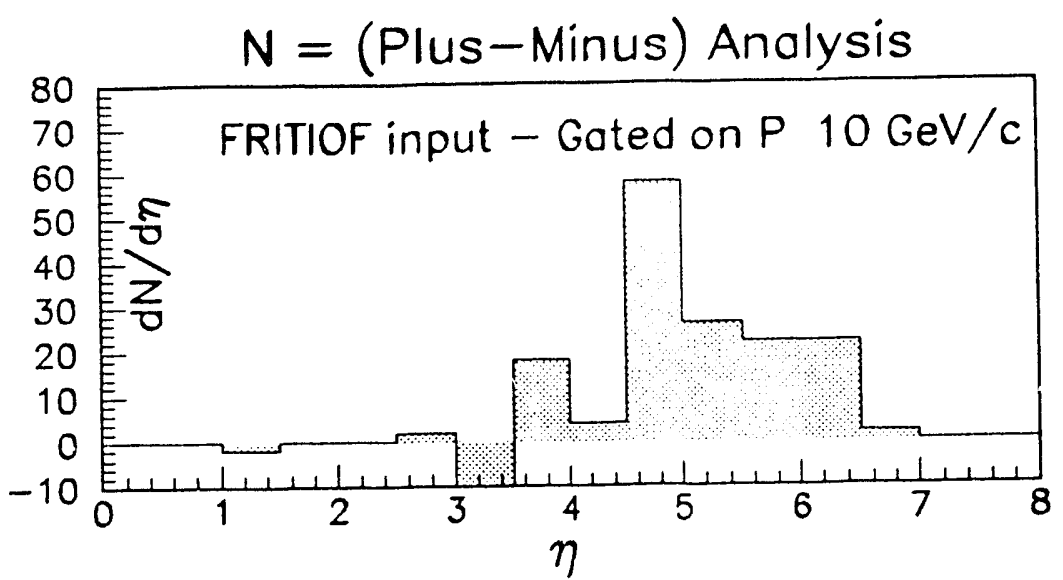

Fig. 14. The upper portion displays the $\eta$ distribution of positive particles minus negative particles. The lower portion of the plot displays the expected response o, an external radial TPC with acceptance $|\eta|<5.5$.

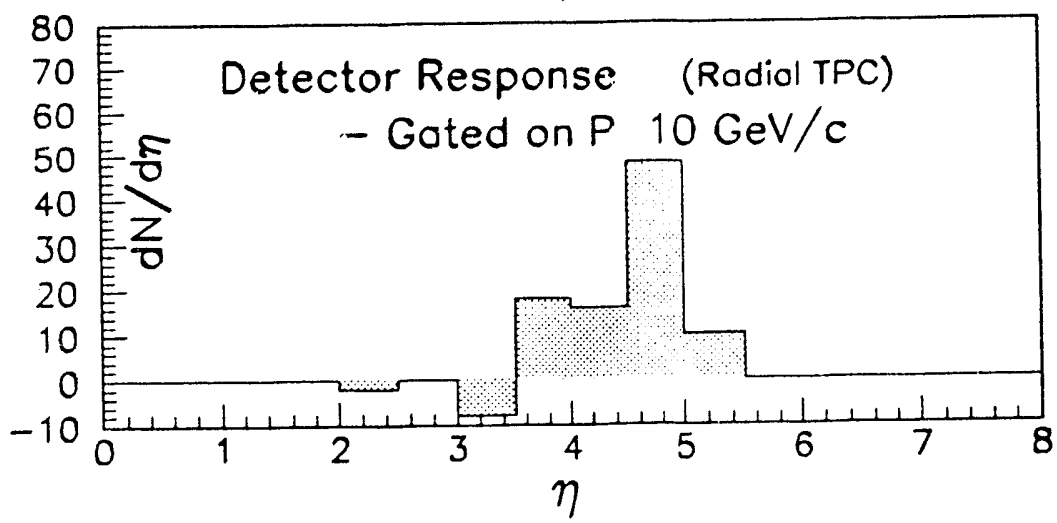

\begin{tabular}{|l|l|}
\hline \multicolumn{1}{|c|}{ Extcrnal TPC Specifications } & \\
\hline \# Sections & 8 \\
\hline Length & $180 \mathrm{~cm}$ \\
\hline Width (drift distance) & $160 \mathrm{~cm}$ \\
\hline Depth & $100 \mathrm{~cm}$ \\
\hline$\#$ pads for each unit & 900 \\
\hline Total number of pads & 7200 \\
\hline Pad size & $1 \mathrm{~cm} \times 20 \mathrm{~cm}$ \\
\hline Tracking accuracy & 250 microns \\
\hline Time samples & 512 \\
\hline Drift time $(3.2 \mathrm{~cm} / \mu \mathrm{s})$ & $50 \mu \mathrm{s}$ \\
\hline
\end{tabular}

\section{Time-of-Flight (TOF) Detectors}

The physics goals of STAR, particularly for complete particle identification, demand a comprehensive TOF system. The requirements for this system are: 1) coverage of the pseudorapidity range $|\eta|<1$ subtended by the barrel surface of the TPC; 2) identification of pions with $0.3<\mathrm{p}_{\mathrm{t}}<1.5 \mathrm{GeV} / \mathrm{c}$, kaons with $0.3<\mathrm{p}_{\mathrm{t}}<1.5$ $\mathrm{GeV} / \mathrm{c}$, and protons/antiprotons with $0.3<\mathrm{p}_{\mathrm{t}}<2.3 \mathrm{GeV} / \mathrm{c}$; and 3) provide a fast trigger sensitive to charged particle multiplicity in the central region.

The technicai requirements posed by these goais are challenging. The timing resolution needed is $\sigma<100 \mathrm{ps}$ and the system must cover a large area. The ner.essity 
of minimizing secondary particles, particularly photon conversions, suggests thai the TOF system should be inside the solenoid coil and cryostat, where the TOF elements must function in a $0.5 \mathrm{~T}$ magnetic field.

Several TOF schemes are presently under investigation. One configuration with two variations is closest to established technology, involving scintillators and photomultiplier tubes (PMTs). This will be discussed in detail below. Another approach involving a multi-layered configuration of scintillators is being studied but will not be reported in this document. Finally, two other ideas are current R\&D projects, fast gas counters and silicon avalanche diodes. Both would deploy arrays of rectangular

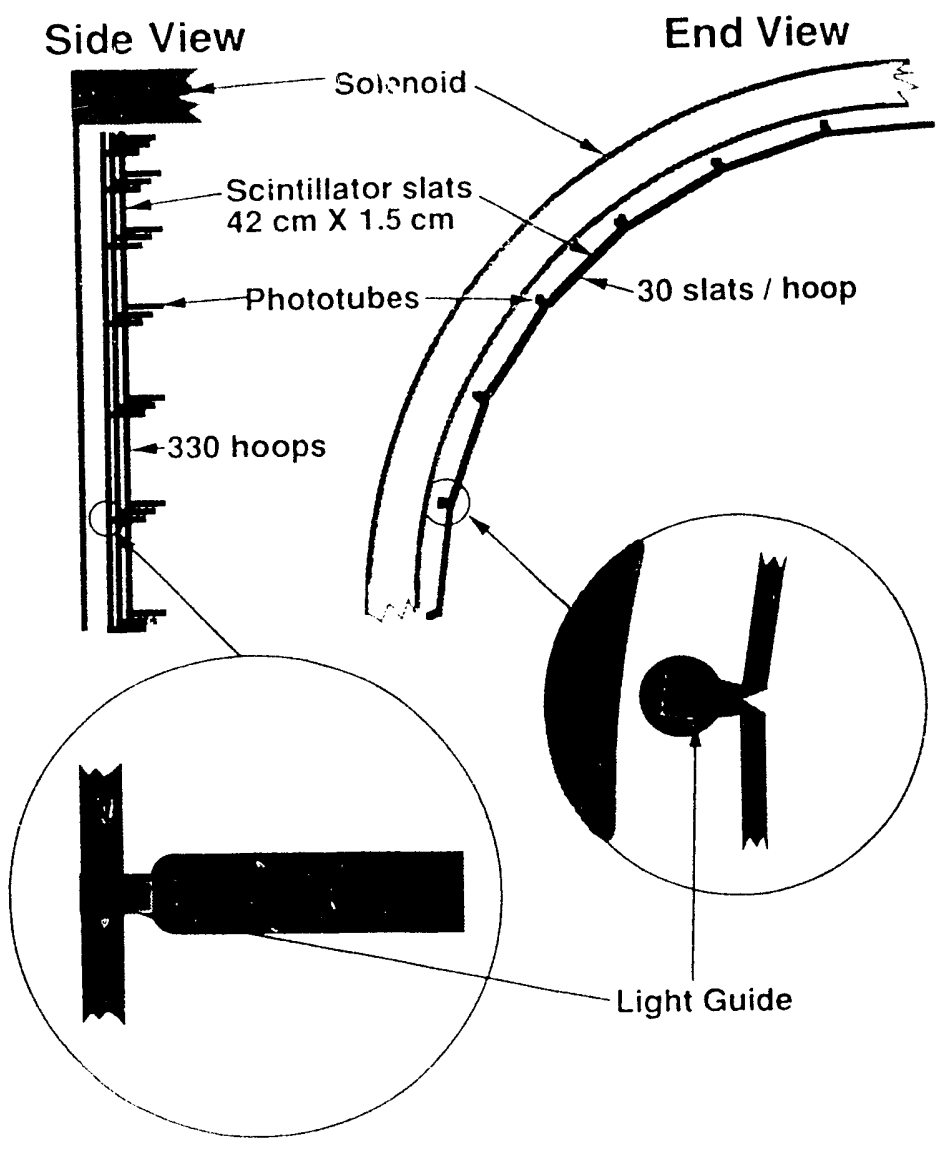

Fig. 15. A schematic of the TOF1 arrangement with the phototubes parallel to the magnetic field. pixels as TOF elements. A brief status report for each is presented in the R\&D summary section at the end of this document.

The most developed idea, using known techniques, consists of compact strips of scintillator inside the solenoidal magnet surrounding the TPC. The TOF table below gives the specifications of the TOF system. Three possible configurations, called TOF1, TOF2 and TOF3, were studied. The TOF1 detector shown in Fig. 15 consists of 330 hoops of TOF elements with 30 TOF slats each $42 \mathrm{~cm} \times 1.5 \mathrm{~cm}$; two adjacent slats are connected to a single PMT at the joint. The TOF2 alternative uses 30 slats $42 \mathrm{~cm}$ $x 3.0 \mathrm{~cm}$ for each hoop and each slat is connected to two PMTs with a total of 165 hoops. The TOF3 uses 15 slats $84 \mathrm{~cm} \times 1.5 \mathrm{~cm}$ with two PMTs per slat and 330 hoop elements for the entire detector. The readout for the slats can be accomplished in two ways. The first uses flexible bundles of clear optical fibers to connect the ends of the slats to conventional one inch diameter PMTs located outside of the magnetic field. Bench tests are currently underway with $5 \mathrm{~m}$ length potted bundles of 100 clad fibers of $2 \mathrm{~mm}$ diameter coupled to scintillators to study the time dispersion and light collection properties. The second method uses newly developed transmission mesh dynode PMTs (Hamamatsu) inside the magnetic volume of the solenoid. The PMTs are arranged such that the axis of the tube is parallel to the magnetic field. These tubes have been shown to perform well for 
magnetic fields up to $1.0 \mathrm{~T}, 25$ with a time resolution better than $100 \mathrm{ps}$ in conjunction with such slat configurations.

\begin{tabular}{|lc|}
\hline \multicolumn{2}{|c|}{ Time-of-Flight Detector } \\
\hline Radius & $2 \mathrm{~m}$ \\
Area & $60 \mathrm{~m}^{2}$ \\
Slat size & $42 \times 1.5 \mathrm{~cm}^{2}$ \\
Channels & 9,900 \\
Time resolution & $<100 \mathrm{ps}$ \\
\hline
\end{tabular}

Some of the particles will have only one good timing measurement, in which case the tracking information will be necessary to resolve the ambiguity in TOF assignment. Several typical Fritiof central Au + Au events were used and combined with a GEANT simulation which included the beam pipe, SVT, TPC and magnet. Approximately 1000 primary and 2000 total charged particles
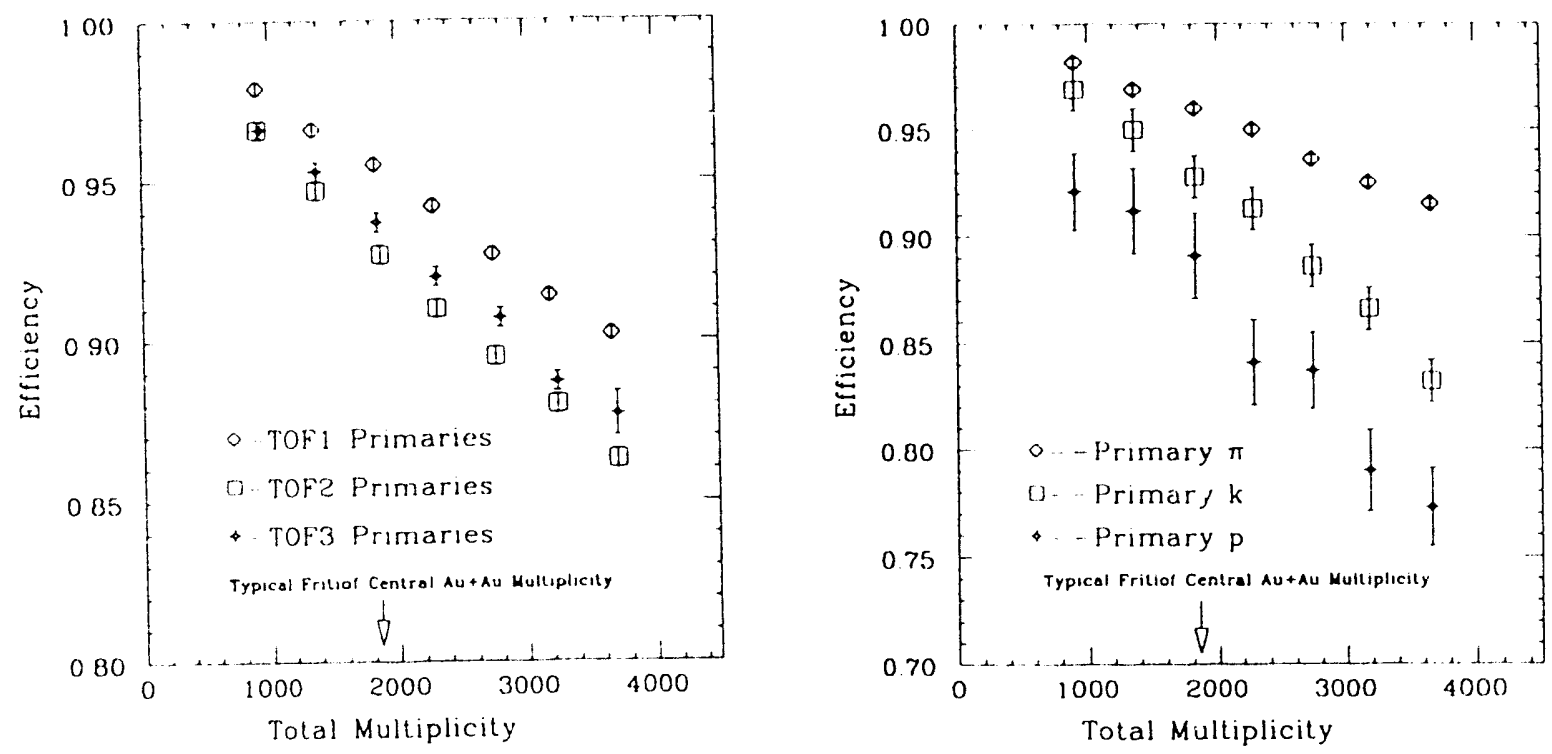

Fig. 16. Mean particle identification efficiency for all particle types as a function of multiplicity for the TOF1, TOF2, and TOF3 arrangements.

Fig. 17. Particle identification efficiency as a function of multiplicity for $\pi, K$, and $p$ for the TOF1 arrangement.

reach the TOF in a typical event. Simulation of the TOF1 system response indicates that $95 \%$ of the primary particles have at least one good timing signal recorded in a PMT. The multiplicity density was varied by merging events or throwing away tracks randomly within the TOF acceptance. Fig. 16 shows a comparison of the percentage of primary tracks with at least one good timing measurement (PID efficiency) as a function of charged particle multiplicity for the TOF1, TOF2 and TOF3 configurations, where primary tracks are defined as those originating from $|z|<1.0 \mathrm{~cm}$ along the beam from the interaction and $|x, y|<0.5 \mathrm{~cm}$ transverse to the beam. Fig. 17 shows the PID efficiency for primary pions, kaons and protons with

25 G. Finsel et al., Nucl. Inst. and Meth. in Phys. Res. A290 (1990) 450-456. 
the TOF1 configuration. When the $\mathrm{dE} / \mathrm{dx}$ from the TPC is used to identify slow particles, the combined PID efficiency is higher for protons, as described in section $F$ below.

We have also performed a simulation with the above TOF configuration(s) outside the magnet coil. This simulation included the effects of secondary hadron production and photon conversions in the material of the coil. The results of this study indicate that the photon conversions and secondary reactions make this configuration impractical.

\section{E. Calorimetry}

\section{EM Calorimeter}

A fast readout calorimeter is necessary as a trigger for the STAR experiment to facilitate rapid selection of events (centrality via $E_{t}$ selection, jets, etc.). A multiplicity trigger can perform some of these functions but at a reduced level. The EM calorimeter design proposed for STAR provides multiplicity data as well as EM exclusive data at the trigger level. Aside from triggering, the type and quality of calorimetry will determine the specific physics that can be addressed by the calorimetry. The best performance, most cost-effective solution appears to be a design which uses $60 \mathrm{~cm}^{2}$ cells with three-fold depth segmentation to efficiently $(95 \%)$ identify EM energy at moderate resolution $(15 \% / \sqrt{ } \mathrm{E})$. This cell size corresponds to using $1.1 \times 10^{4}$ towers surrounding the coil of the solenoid. This EM calorimeter covers a pseudorapidity range of $|\eta|<1.05$. The addition of a wire chamber with better shower position resolution is under study, for $\gamma / \pi^{\circ}$ discrimination in measurements with polarized proton beams and possibly heavyions. The design discussed in the LOI has been eliminated as a candidate for a manydepth or finely segmented device due to this lack of hermeticity and a high readout cost with conventional phototubes. As a result of a design study for a stand alone EM calorimeter which will allow EM energy identification, it is concluded that only two types of calorimeters would be effective from a physics and a cost standpoint. The first is a design based on a liquid argon calorimeter with readout costs of about $\$ 250 /$ channel. The second uses WLS optical fibers reading out plastic scintillator plates or "tiles". The design developed by members of the STAR collaboration uses one fiber per tower or tower depth segment, thus concentrating the light output into a single $1.5 \mathrm{~mm}$ optical fiber. This allows a hermetic design and cheap readout at $\$ 40-45 /$ channel with segmented anode phototubes. The EM calorimeter is a compact shell with either technology. The energy resolution of both is determined principally by sampling fluctuations, i.e. the plate thickness for dividing up the 10.5 $\mathrm{cm}$ of lead absorber.

\section{Hadron Calorimeter}

The magnet design allows for the inclusivii of a hadronic calorimeter between the EM calorimeter and the outer yoke. For reasons of cost, this detector is expected to be staged and is not included in the initial cost estimate. 


\section{F. STAR Capabilities}

\section{Acceptances for Tracking and PID}

The acceptance for STAR has been calculated using GEANT to simulate charged particle hits on the proposed pad plane configuration and a tracking code for event reconstruction. The acceptance for tracking in the TPC is shown in Fig. 18. The outer, solid histogram is the distribution for all charged particles from the primary vertex for central $\mathrm{Au}+\mathrm{Au}$ collisions. The dashed histogram is the $\eta$-distribution for all charged primary vertex tracks which cross at least five pad rows in the TPC. The

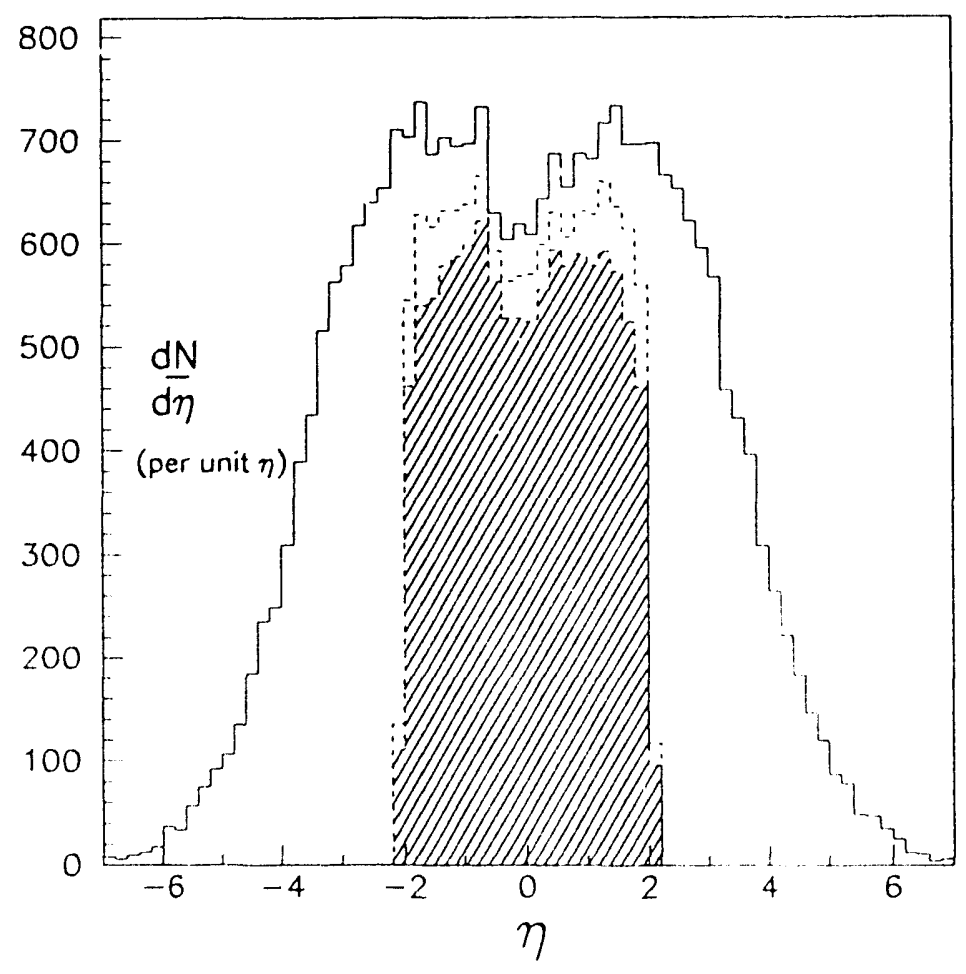

Fig. 18. The tracking acceptance as a function of $\eta$. Outer solid histogram is all charged tracks input to GEANT simulation. Dashed histogram is charged primary vertex tracks which cross $>5$ pad rows in the TPC; filled cross-hatched histogram is tracks found and reconstructed. difference in the central region is due to decays and interactions of primaries. The inner crosshatched histogram is the $\eta$-distribution for the charged primary vertex tracks found by the tracking software. For a track to be accepted, $90 \%$ (i.e., $\mathrm{R}=0.9$ ) or more of the hits associated with the track must correspond to the correct GEANT track.

Two features shown in Fig. 18 should be emphasized. First, the acceptance of the STAR detector configuration quoted in the past was underestimated. Comprehensive tracking simulations have shown that the tracking acceptance of STAR is highly efficient over $|\eta|<2$. Second, the figure illustrates the high tracking efficiency of the TPC in the RHIC environment even in the presence of secondaries from decays and interactions of primaries. Note that the simulated event was generated with the detector and beam line materials included and all physics processes activated in GEANT. There are approximately equal numbers of primary and secondary particles.

The particle identification (PID) capabilities of STAR, including the effects of the tracking acceptance and efficiency, have also been investigated. The PID via $d E / d x$ in the TPC gas and/or via time of flight (TOF) is calculated on a track-by-track basis in GEANT. For PID via $\mathrm{dE} / \mathrm{dx}$ a $3 \sigma$ separation is required. For PID via time-of-flight at least 300 ps difference is required between the 'ime-of-flight of the most probable species $(\pi, K, p)$ and the next most probable. 


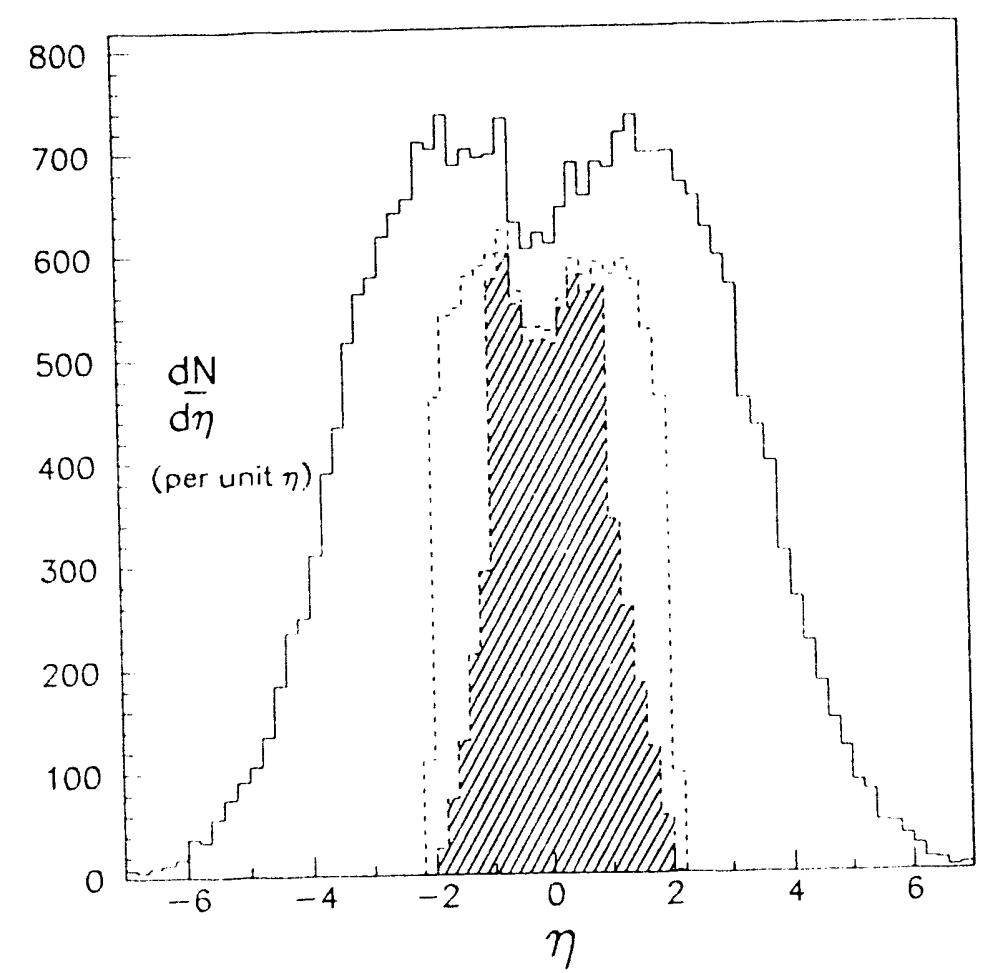

Fig. 19. Particle identification capabilities in terms of $\eta$. Outer solid histogram is all charged tracks input to GEANT simulation. Dashed histogram is found tracks. Cross-hatched histogram is found tracks for which PID is possible.
Fig. 19 shows the PID acceptance in terms of $\eta$. The outer, solid histogram is the $\eta$ distribution of all charged tracks from the primary vertex. The dashed histogram is the same as the filled histogram of Fig. 18, representing the tracks successfully reconstructed by the tracking software. The cross-hatched histogram is the $\eta$ distribution for the found tracks for which one expects to achieve PID.

\section{HBT}

The maximum source radius that can be meaningfully investigated in the STAR experiment is limited by the radial (outward) momentum resolution of the system. Analysis of the STAR systems shows that sources with $R_{t}=$ $10 \mathrm{fm}$ can be investigated with bosons of momenta up to 800 $\mathrm{MeV} / \mathrm{c}$, sources with $R_{t}=20 \mathrm{fm}$ with bosons up to $600 \mathrm{MeV} / \mathrm{c}$, and $\mathrm{R}_{\mathrm{t}}=40 \mathrm{fm}$ with bosons up to 400 $\mathrm{MeV} / \mathrm{c}$, which is larger than the $\left\langle\mathrm{p}_{\mathrm{t}}\right\rangle$ of the predicted pion momentum distribution. Thus the STAR detector should be well suited for pion HBT interferometry.

The HBT capabilities with kaons will be more limited with STAR because the kaons are expected to be a factor of 10 lower and have a momentum distribution which peaks at about $400 \mathrm{MeV} / \mathrm{c}$. Even with these restrictions, however, ensemble kaon HBT interferometry with STAR will permit investigation of the size and duration of the source at the time when kaons are emitted, a significantly earlier era in source evolution than the era of pion emission.

The Silicon Vertex Tracker (SVT) of the STAR detector system leads to important improvements in HBT capabilities. The SVT extends the capabilities of the system in the low momentum region, reduces sensitivity to multiple scattering in the TPC walls, and improves transverse and longitudinal paired-particle momentum resolution at all momenta. The fact that the STAR detector has equal acceptances for positive and negative particles also offers a powerful advantage for HBT, because $(++)$ and (--) HBT correlations can be compared or combined for better statistics, and (+-) correlations and possibly larger numbers of mixed charges ${ }^{26}$ can be used as a test of the Coulomb corrections used in the analysis.

26 J.G. Cramer, Phys. Rev. C43 (1991) 2798. 


\section{Fluctuation Analysis}

\section{Long Range Fluctuations}

The addition of tracking multiplicity detectors at high $\eta$ has enhanced the STAR detector's capability to observe the fluctuations in multiplicity and transverse energy that may be characteristic of a phase transition. These fluctuations may occur at many length scales, in a pseudorapidity interval as large as one unit or more. It is important that the detector cover the full azimuth in order to distinguish between fluctuations in $\eta$ and $\phi$ on the one hand and enhanced but uniform particle or transverse energy production on the other. As an example, $\mathrm{dn} / \mathrm{d} \eta$ and $\mathrm{dn} / \mathrm{dy}$ distributions are shown in Fig. 20 for a special event ${ }^{27}$ with a long range fluctuation. The fluctuation in Fig. 20 can be seen in all particle channels and can be identified via $\mathrm{dn} / \mathrm{d} \eta$ as well as $\mathrm{dn} / \mathrm{dy}$ distributions. A detailed study of the flavor content of the fluctuation can be made over $\Delta y=2$ at midrapidity in STAR.
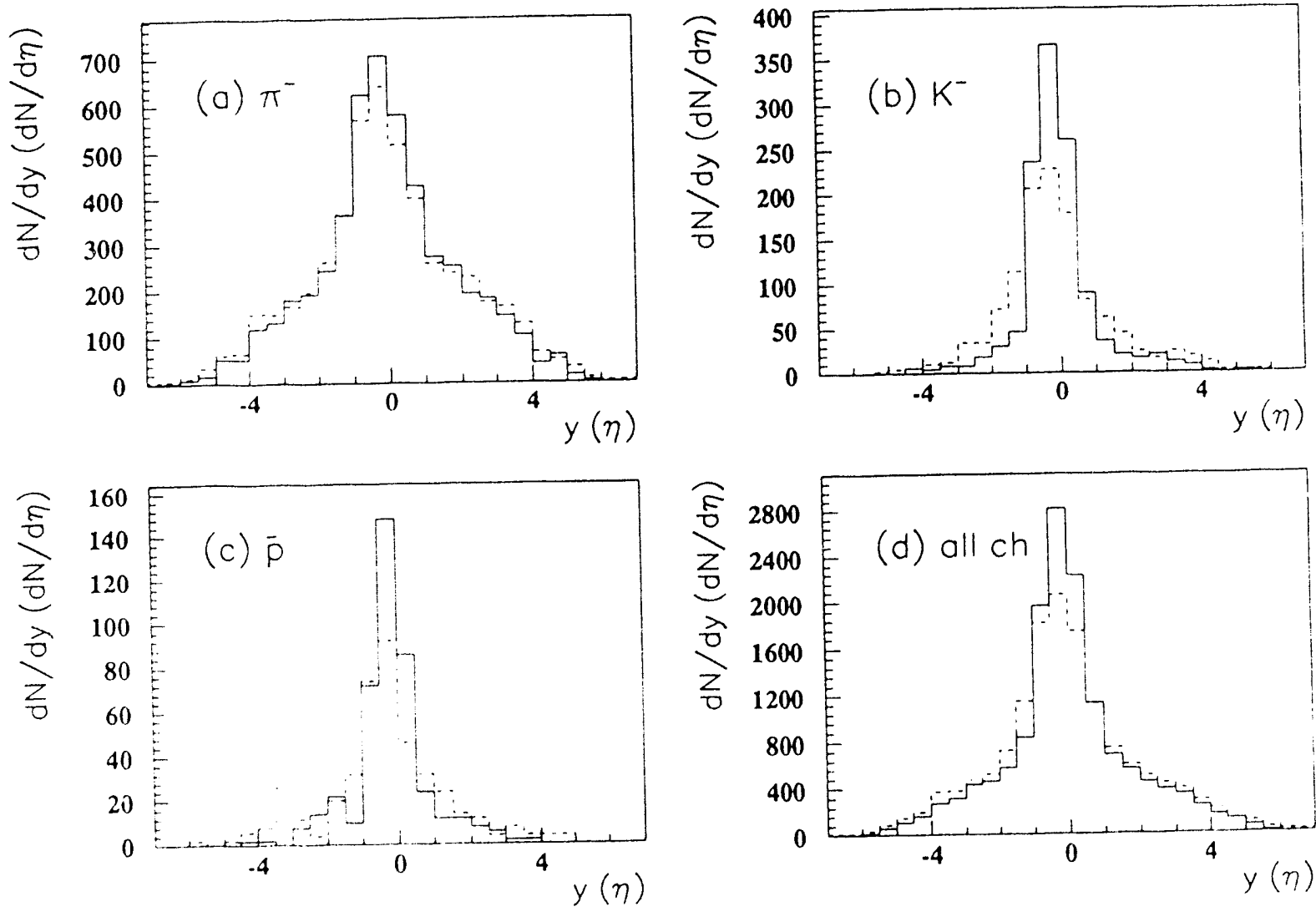

Fig. 20. $d N / d y$ (solid) compared to $d N / d \eta$ (dashes) for a single central $A u+A u$ RHICPLASMA event, 27 plotted for (a) negative pions, (b) negative kaons, (c) antiprotons, and (d) all charged particles. No corrections have been made for acceptance or tracking efficiency. The large bump near $y=0$ in all distributions corresponds to particles produced mainly from a plasma bubble.

27 G. Danby et al., RHIC Letter of Intent \# 3 (1990). 


\section{Electromagnetic/Hadronic Energy Balance}

Cosmic ray experiments have found events with unusual ratios of electromagnetic to hadronic energy. There are only a few such events, and various theoretical explanations have been proposed, usually incorporating the QGP. ${ }^{11}$ In the STAR detector, the EM Calorimeter combined with a fast charged-particle multiplicity detector (the TOF array), will allow a simple, fast trigger to collect many such events and study their properties.

\section{Short Range Fluctuations (Intermittency)}

Intermittency, or Factorial Moment Analysis ${ }^{28}$, is a powerful technique for studying details of the charged particle multiplicity distribution, and is sensitive to multiparticle correlations over many length scales in phase space. There are trivial correlations in the multiplicity distribution due to, for instance, resonances, and in order to uncover new physics it is necessary to probe the distribution at resolutions in $\eta$ and $\phi$ well below the scale $(\Delta \eta \sim 0.5)$ characteristic of these trivial correlations. In the STAK detector, the displacement due to multiple scattering is equal to the twotrack separation in the SVT for $110 \mathrm{MeV}$ pions and $205 \mathrm{MeV}$ kaons; at the outer radius of the TPC it is equal for $210 \mathrm{MeV}$ pions and $400 \mathrm{MeV}$ kaons. Thus, the finest bin size for intermittency analysis is governed primarily by the two-track resolution, either of the SVT or the TPC.

At midrapidity, the Jacobian $\Delta \eta \Delta \theta=1$, so that the bin size in rapidity can be calculated directly from the two-track resolution. For the SVT, a two-track resolution of 750 microns, and a distance of about $10 \mathrm{~cm}$ from the interaction are assumed. This gives bin sizes of $\Delta \eta=\Delta \phi \sim 0.0075$. For the TPC, we estimate a twotrack resolution of $2 \mathrm{~cm}$ at a distance of $2 \mathrm{~m}$, giving bin sizes of $\Delta \eta=\Delta \phi \sim 0.01$. These capabilities, as well as the STAR pseudorapidity coverage, match or exceed those of current collider detectors performing intermittency analyses. ${ }^{29}$ In addition, the proposed EM calorimeter (cell size $\sim 8 \times 8 \mathrm{~cm}^{2}$ ) will have a minimum bin size of $\Delta \eta$ $=\Delta \phi \sim 0.04$. Because of its PID capability through longitudinal segmentation, it will offer the unprecedented opportunity of performing high resolution intermittency analysis on $\pi^{0}$ s and photons.

A recent publication ${ }^{30}$ reported an intermittency analysis of the partition of full three-dimensional Lorentz Invariant Phase Space. The STAR detector will momentum analyze virtually all charged particles within $|\eta|<2$, so that this type of intermittency analysis should be possible at STAR. However, the resolution of the momentum difference between two tracks (which governs the binning in the third dimension) is a complex matter, and the capability to do this has yet to be determined for STAR.

\section{Jet Studies Using EM + Tracking}

One of the major changes made in the proposed detector configuration since the LOI is the staging of the hadronic calorimetry, on the basis of cost. However, it

28 A. Bialas and R. Peshansky, Nucl. Phys. B273 (1986) 703.

29 UA1: Nucl. Phys. B34 (1990) 1; DELPHI: Phys. Lett. B247 (1990) 137; OPAL: CERN-PPE/91-37.

30 CELLO: Phys. Lett. B256 (1991) 97. 
should be possible to make many of the desired measurements of jets and high- $\mathrm{p}_{\mathrm{t}}$ particles by combining the information from tracking and electromagnetic (EM) calorimetry alone. There are two major issues to be addressed: 1) how significant is the loss in jet energy resolution caused by the fluctuating losses of long-lived neutral hadrons (neutrons and $\mathrm{K}_{\mathrm{L}} \mathrm{L}$ ), and 2) how one provides an adequate trigger for the events of interest.

The first question was studied by simulating jet events in pp collisions at $\sqrt{ }_{s_{n n}}=$ $200 \mathrm{GeV}$ using the HIJING generator, decaying all produced particles with lifetimes

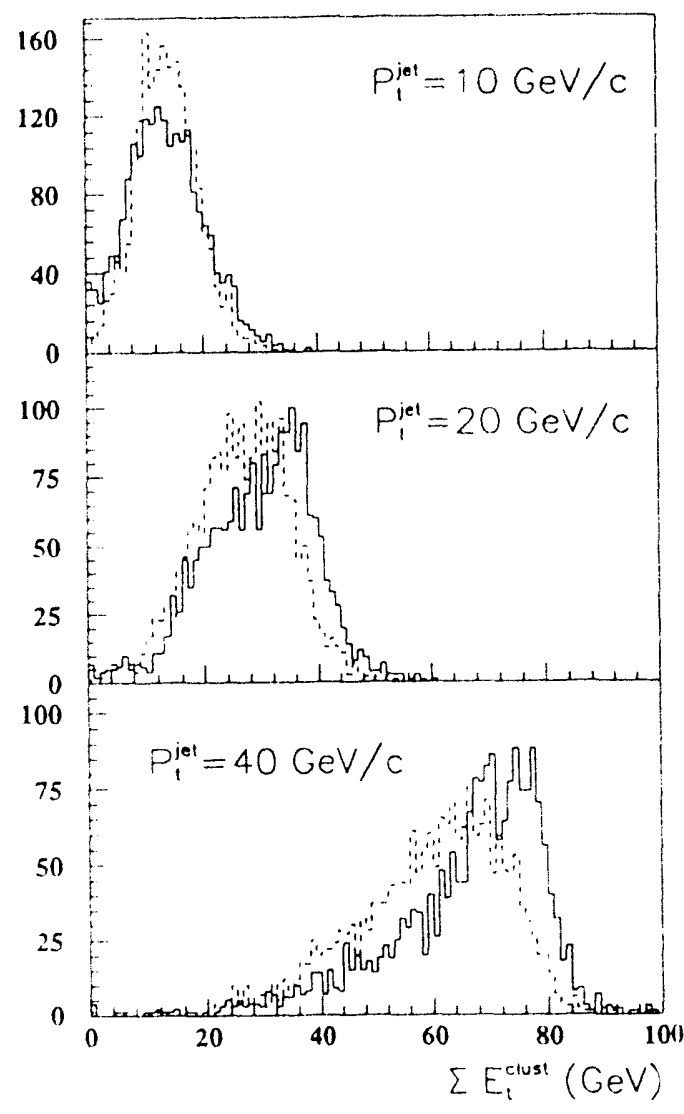

Fig. 21. Et for jets of 10, 20, and 40 $\mathrm{GeV} / \mathrm{c}$. The solid histograms are for EM plus hadronic calorimetry and the dashed histograms are for EM plus tracking. shorter than the $\mathrm{K}^{\circ} \mathrm{L}$, and considering the energy produced in the form of photons, charged particles and hadrons (both charged and neutral). The effect of the STAR solenoidal field on the location of the energy deposited by the charged particles in the hadronic calorimeter was taken into account, and then the energies of the hadrons and photons were binned into an $\eta-\phi$ grid with bin sizes of 0.1 and 0.028 , respectively. (These bins are much larger than necessary for the 'Tracking' and EM components, but are used for comparison with the bin sizes typical of collider calorimeters.) The resulting binned energies were folded with resolutions of $0.40 / \sqrt{ } \mathrm{E}$ for the hadron calorimeter, and $0.22 / \sqrt{ } \mathrm{E}$ for the $E M$ calorimeter. The spatial resolution, momentum resolution, and reconstruction efficiency of the tracking system have so far been assumed to be perfect. Our implementation of the CDF cluster-finding aigorithm was then applied to the binned data sets and, for each found cluster, $E_{t}$ was summed within $R=\sqrt{ }\left(\Delta \eta^{2}+\Delta \emptyset^{2}\right) \leq 0.7$ for both the [EM + hadronic] and [EM + Tracking] data sets. A grand sum over all clusters found in each event was then made in both cases. A comparison of the two types of 'calorimetry' is shown in Fig. 21, for jet $\mathrm{p}_{\mathrm{t}}$ 's of 10,20 and 40 $\mathrm{GeV}$. Note that the energy displayed is the total contained within the 'calorimeter' coverage, $|\eta|<1.05$, and would be equal to twice the one-jet $p_{t}$ if it were not for energy escaping through the ends of the calorimeter barrel. This loss of contained two-jet energy can be significantly reduced by rejecting events which deposit significant 'Tracking' energy within $1.05<|\eta|<2.0$ (as discussed in the following section). The mean detected jet energies and fractional RMS widths for these data are listed in the table below. 


\begin{tabular}{|c|c|c|c|}
\hline Et (jet) & $20 \mathrm{GeV} / \mathrm{c}$ & $40 \mathrm{GeV} / \mathrm{c}$ & $80 \mathrm{GeV} / \mathrm{c}$ \\
\hline EM + Hadronic & & & 65.32 \\
\hline mean detected & 13.77 & 29.70 & 0.65 \\
\hline detected/generated & 0.83 & 0.65 & 13.99 \\
\hline RMS width & 6.60 & 9.59 & 14.5 \\
\hline \% RMS width & 40 & 24 & 59.01 \\
\hline EM + Tracking & & & 0.79 \\
\hline mean detected & 13.98 & 26.79 & 13.50 \\
\hline detected/generated & 0.81 & 0.76 & 17 \\
\hline RMS width & 5.21 & 8.49 & 25 \\
\hline \% RMS width & 32 & 24 & \\
\hline
\end{tabular}

The energy resolutions in [EM + Tracking] are not appreciably worse than those in [EM + Hadronic] for the higher $p_{t}$ jets, and are even somewhat better for the lowest $p_{t}$, presumably because of the rapidly decreasing hadronic calorimeter resolution.

Studies of the ability of the EM calorimeter to deliver a trigger for events containing jets or several high- $\mathrm{p}_{\mathrm{t}}$ particles have begun with the simulations of $\mathrm{pp}$ events as described above. Calculations were performed for two possible triggers,

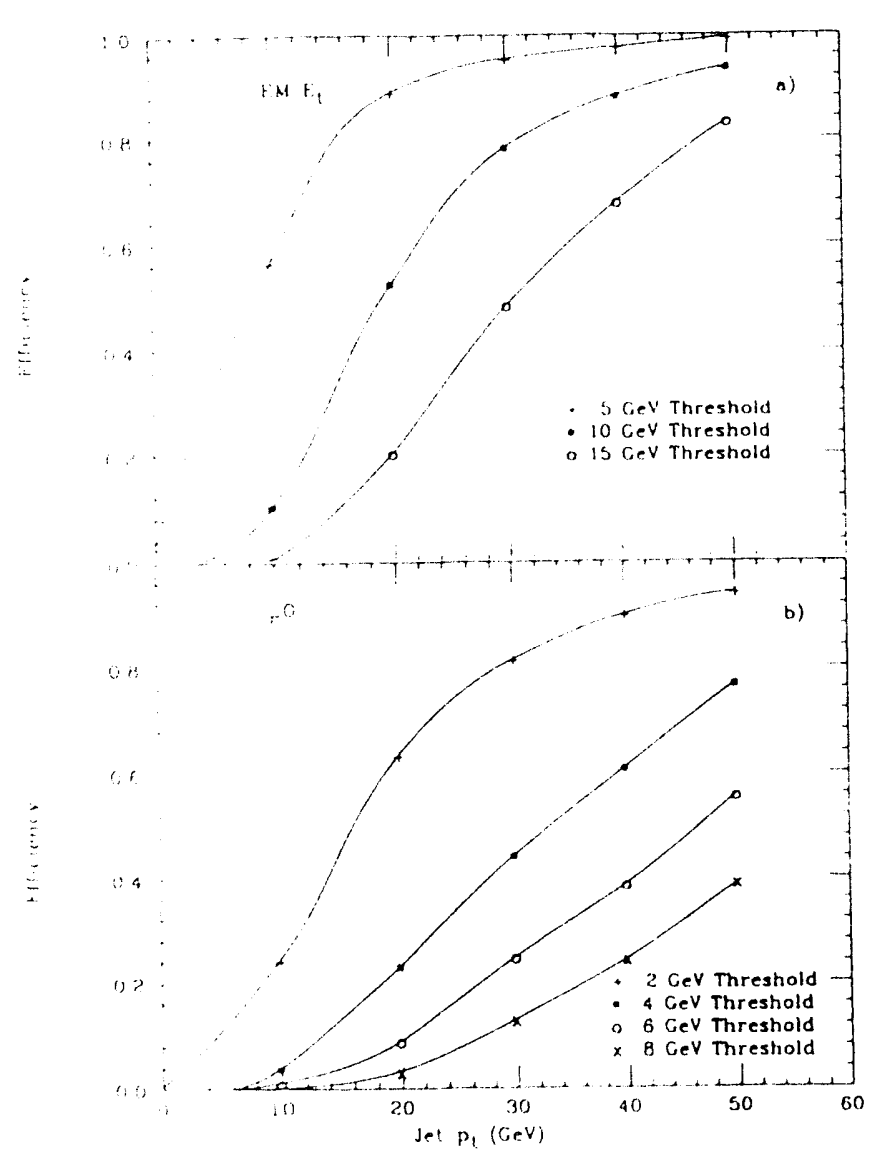

Fig. 22. Trigger efficiency versus jet energy for different thresholds. a) is for EM energy and $b$ ) is for $\pi^{\circ}$ energy. one based on total $E_{t}$ within the EM calorimeter and a second based on a single high-p $\pi_{t} \mathrm{o}$. The efficiencies of these triggers are shown vs jet $p_{t}$ in Fig. 22.

\section{$E_{t}$ Resolution Effects Due to Finite} Acceptance

One aspect of the STAR jet physics capability at RHIC which is presently under study is the EM/tracking coverage in pseudorapidity necessary to insure that fluctuations in $\mathrm{E}_{\mathrm{t}}$ due to losses outside the region of coverage are no larger than those due to missing neutral energy (neutrons, $\mathrm{KO}_{\mathrm{L}}$ ) which will not be detected. A preliminary study of this aspect is illustrated in Fig. 23. The distribution plotted in Fig. 23 is the per event imbalance in $E_{t}$ for di-jets of $80 \mathrm{GeV}$ total energy resulting solely from the loss of jet fragments falling outside the region of coverage. In Fig. 23a, this distribution has been calculated assuming full EM calorimetry and tracking coverage in the region $|\eta|<$ 
1.35. In this instance approximately $10 \%$ of the events result in an $E_{t}$ imbalance of 10 $\mathrm{GeV}$ or more. Fig. 23b illustrates the improvement in this distribution if the same coverage were extended to $|\eta|<2$. The number of events having an $E_{t}$ imbalance of

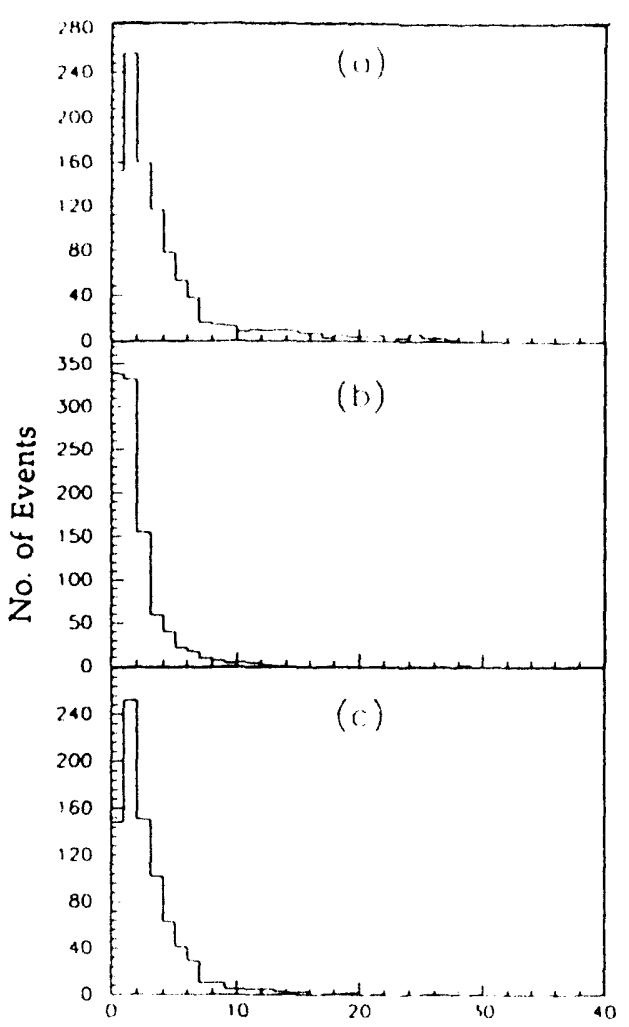

Missing Transverse Energy (GeV)

Fig. 23. The distribution of the per event imbalance in transverse energy $\left(E_{t}\right)$ from $p p$ collisions at $200 \mathrm{GeV}$ containing $40 \mathrm{GeV}$ jets a) assuming full EM and tracking coverage for $|\eta| \leq$ $1.35, b$ ) assuming full $E M$ and tracking coverage for $|\eta| \leq 2$, and c) assuming full EM and tracking coverage for $|\eta| \leq 1.35$ with the additional requirement that the total tracking $E_{t}$ in the surrounding guard region described in the text be less than $5 \mathrm{GeV}$. more than $10 \mathrm{GeV}$ in this case is reduced to $1.8 \%$. For low multiplicity events a "guard region" can be used to minimize the effects of fluctuations due to limited coverage. Events having $E_{t}$ values exceeding an appropriate threshold in the guard region are rejected upon the assumption that for these events an excessive fraction of the reconstructable $\mathrm{Et}$ has been missed due to leakage. Fig. 23c illustrates the improvement when the imbalance in $E_{t}$ is calculated assuming $|\eta| \leq 1.35$, but events having more than $5 \mathrm{GeV}$ of tracking $E_{t}$ in the guard region, $1.35<|\eta|<2$ has been rejected. Although this results in a reduction of approximately $15 \%$ in the number of events sampled, the distribution for the remaining events approximates that for a calorimeter covering a full 4 units of pseudorapidity $|\eta|<2$, only $3 \%$ of the remaining events having an $\mathrm{E}_{\mathrm{t}}$ imbalance of greater than $10 \mathrm{GeV}$.

\section{Computing and Data Acquisition}

The table below lists the major detector components along with the estimated data volume per event (central $A u+A u$ ) and the number of cpu cycles required for event reconstruction (millions of floating point operations). The TPC is by far the largest component and will dominate the data acquisition and computing requirements. 


\begin{tabular}{|l|c|c|}
\hline Detectors & $\begin{array}{c}\text { MB/event } \\
\text { (central Au+Au) }\end{array}$ & $\begin{array}{c}\text { Event Reconstruction } \\
\text { (MFLOP/event) }\end{array}$ \\
\hline TPC & 8 & 4000 \\
\hline TOF & 0.008 & 4 \\
\hline SVT & 0.25 & 125 \\
\hline External TPCs & 0.5 & 250 \\
\hline Calorimeters & 0.004 & 2 \\
\hline Total & 8.8 & 4400 \\
\hline
\end{tabular}

The dominant factor in the cpu requirements for event reconstruction is in processing the pad amplitude information information for hit finding prior to track finding. The hit finding procedure can be performed online ard investigation of the possibilities of doing these calculations within the acquisition system will continue.

For a trigger rate of $10 \mathrm{~Hz}$ for central $\mathrm{Au}+\mathrm{Au}$ events there are considerable bandwidth requirements and a step toward addressing this issue is an R\&D proposal which has been submitted to investigate Futurebus+.

The offline computing requirements are beyond current technology but the rapid pace of developments in the private sector regarding cpu speeds, data storage capacities and network bandwidth are expected to be adequate by the time of RHIC.

\section{Staging}

As discussed above, it is necessary to stage the hadronic calorimeter in order to keep the cost estimate close to $\$ 30 \mathrm{M}$. Although this will have a serious impact on STAR, we believe an initial configuration lacking this component will address the key physics goals of the Collaboration. Further staging may have to be considered. We feel that detailed analyses of these decisions is premature. However, it is clear that staging the magnet, the main TPC, the SVT, or the external TPCs will have very serious consequences for the capabilities of the detector.

\section{Updated Cost Estimate}

The estimated total cost for the proposed experiment is $\$ 30.8 \mathrm{M}$. An outline of the cost estimate is tabulated below. $300 \mathrm{~W}$ of He refrigeration is required for cooling the superconducting coils of the magnet and is assumed to be available from the RHIC cooling system. ${ }^{31}$

31 T. Ludlam, Memorandum, 8 July 1991. 


\section{STAR Cost Estimate}

Magnet

yoke

coil and cryostat

engineering

Silicon Vertex Tracker

Time-Projection Chamber

field cages

sectors and support structure

laser calibration system

electronics (150 K chan.)

Time-of-Flight

Calorimeters EM $(0.6 \mathrm{Ll})$

High $\eta$ Detectors

Computers

Triggering

Total

The cost of the STAR experiment could be reduced if an existing superconducting magnet with similar dimensions were to become available. There may also be calorimetry in existing experiments that is suitable for implementation in STAR.

\section{Engineering Cost for the STAR Proposal}

The cost of engineering to develop a proposal for the STAR experiment is detailed in Appendix B. Included in Appendix B are the following:

a) A cost estimate for the mechanical and electronics engineering effort during the 1st half of FY 92 towards the STAR proposal.

b) A preliminary estimate of the STAR construction schedule.

c) A preliminary estimate of the STAR installation schedule.

d) A list of questions, for RHIC and BNL management, some of which affect the cost and schedules in Appendix B.

\section{RHIC R\&D Update}

\section{A. TPC Electronics}

The main goal of the RHIC R\&D program for integrated TPC electronics is to develop an integrated shaping amplifier with time constants that are reproducible within a few percent. This can be achieved by tuning differentiation and integration stages with correction capacitors that are switched with fuses. Several integrated circuits with tunable integrators, differentiator and fuses have been developed and constructed. An integrated four-channel tunable shaping amplifier is presently being fabricated in CMOS technology and will be ready by the end of July. Work continues in parallel to combine the shaping amplifier with the EOS low noise preamplifier and to develop a 512 channel switched capacitor array. 


\section{B. SVT Simulations}

Substantial progress has been made in FY 91 in the SVT RHIC R\&D project towards understanding the ability of the SVT including: 1) improving the main vertex resolution, 2) matching tracks with the TPC and 3) detection of strange particles $\left(\mathrm{K}^{\circ}, \Lambda, X\right)$ via their charged particle decay modes and determination of the efficiency for detecting them in the STAR setup. Considerable time and energy has gone into establishing the necessary software programs (FRITIOF/GEANT/tracking algorithms) to carry out this task. This has allowed us to investigate the desired geometry for the SVT and its integration with other STAR systems. For FY 92 we will be concentrating on the following: 1) inclusion of full detector response and effects of smearing of TPC tracks and SVT hits on overall efficiencies and resolutions, 2) we expect to test a prototype silicon drift chamber and would incorporate our experiences with it into the simulation package, 3) investigation of advanced computer techniques such as the Kalman filter method which has been successfully used at LEP to improve pattern recognition and tracking finding, 4) establish whether the SVT arrangement together with track matching to the TPC can detect the production of D-mesons in the high multiplicity environment of RHIC and, 5) optimize the geometry.

\section{Avalanche Readout TPC}

Supported by DOE/ Nuclear Physics funds for generic R\&D, a group led by Dave Nygren of LBL is studying the sparking problem in avalanche detectors so that they can be used on the pad plane of a TPC. If successful this would greatly improve the two-track resolution allowing better HBT measurements and readout closer to the beam line. In collaboration with the Weizmann Institute tests of sparking suppression will be made of a conductive Kevlar filament, an insulating mesh, and a semi-insulating coating on the anode. A test bench has been set up for this work.

\section{Calorimetry Readout}

Supported by DOE/Nuclear Physics funds for generic R\&D, the development of a sampling calorimeter readout scheme based on wavelength shifting optical fibers has resulted in some significant improvements and has allowed cost-effective construction of hermetic highly segmented devices. Through proper matching of the emission properties of plastic scintillators to the fiber collection properties, rather high light levels have been obtained in our work. Typically 8-10 photoelectrons per plate have been measured for $1.5 \mathrm{~mm}$ fiber bonded into $3 \mathrm{~mm}$ thick scintillator sheet, with good uniformity of response. The bonding of one fiber per scintillator plate in a parallel edge geometry was mass produced in the Participant Calorimeter project completed in 1988, similar to current HEP development of the WLS fiber method. These high light level techniques have been applied to an old idea of perpendicular fiher geometry relative to scintillator tiles. Readout of an entire tower with a single optical fiber allows a hermetic design, fine segmentation, and the use of small cost-effective active devices for readout, e.g. photodiodes, segmented anode PMT's, etc. A generic detector development project has produced a fine-grained 100 tower EM test section consisting of $2 \mathrm{~mm} \mathrm{~Pb}$ and 3 $\mathrm{mm}$ scintillator totaling 25 radiation lengths and read out with a single $1.5 \mathrm{~mm}$ 
WLS fiber per tower. The response across the test section is uniform to a few per cent due chiefly to the method used to obtain a high light output of 5-6 pe/plate for a mip. Besides proving the point that a single fiber can be used to reaciout an entire tower of a rather high resolution sampling calorimeter (and with uniform response) the extreme concentration of light in this manner allowed the successful application of 256 channel segmented anode PMT's. Longitudinal segmentation of the calorimeter for PID was accomplished with partially aluminized fibers. Further development and prototyping is needed to obtain a more uniform response for the relatively large towers $\left(60-70 \mathrm{~cm}^{2}\right)$ in the STAR EM calorimeter. Presently, bonded fibers in $40 \mathrm{~cm}^{2}$ plates give satisfactory unifo' $\mathrm{m}$ response at the 3-4 pe level for a mip, but a large amount of construction cost would be saved if fiber bonding were not necessary (which approximately doubles the light output compared to an air gap). Similaily, work is needed on casting techniques for plastic scintillator and for $\mathrm{Pb}$-alloys and $\mathrm{Pb}-\mathrm{W}$ composites in order to minimize machining costs.

\section{E. Silicon Avalanche Diodes}

The Purdue University and LBL groups are investigating silicon avalanche diodes as time-cf-flight detectors. Thin ( $0.15 \mathrm{~mm}$ thick) silicon $\mathrm{n}$-p-i diodes will be exposed directly to fast charged particles, and the internal electric field in the diodes will collect electrons and holes deposited in the silicon by $\mathrm{dE} / \mathrm{dx}$. The avalanche region at the diode junction will give a current gain of 20 to 50 , and a series of fast amplifiers will boost the signal by another factor of 1000 . The resulting current pulse will have a risetime of about $1 \mathrm{~ns}$, duration of $5 \mathrm{~ns}$, and amplitude of $0.5 \mathrm{~V}$. These signals will be discriminated, and fed into a TDC and ADC. The detectors are very low mass, and are insensitive to 1 Tesla magnetic fields. Custom diode assemblies have been purchased from EG\&G ElectroOptics Division, Montreal. These diodes are $0.5 \times 0.5$ square $\mathrm{cm}$. Tests are being conducted at Purdue with two diodes in coincidence, exposed to a beta source. The basic result will be a time resolution histogram from timing one diode against the other. Time resolution of order $100 \mathrm{ps}$ is anticipated.

The longer term R\&D plan is to develop large area arrays of diodes to make detectors of a useful size. If technical feasibility is established in the lab, a program studying diode fabrication, gain uniformity, yield, and cost reduction will begin. Radiation hardness will also be evaluated.

\section{F. Integrated TOF Electronics R\&D}

G. Rai of LBL, in collaboration with the Purdue University Group, is investigating integrated TOF electronics. Development of integrated electronics is vital for any TOF system at RHIC, regardless of the detector technology (e.g. scintillator/PMTs, silicon avalanche diodes, or fast gas counters). Such electronics will be as important to the 2,000 to 20,000 TOF channels as they are to the 150,000 channels of the TPC. Each channel will have an amplifier, discriminator, TDC, and ADC. There must also be supporting electronics to read out the channels, suppress zeroes, and multiplex the signals to the computer. At LBL, 6 jit GaAs TDC chip is presently being tested. RHIC R\&D funds will be requested in fiscal 1992. Once this 
effort receives support, design and prototype testing of custom circuits will begin in collaboration with engineers at LBL, BNL, and industry.

\section{G. Fast Gas Counters for TOF}

The possibility of using high pressure gas counters to achieve low cost 100 ps time-of-flight detectors is being investigated by a group led by Chuck Gruhn. This project is supported by the L.BL Director's Program Development Funds. Both smallgap parallel plate and wire counters are being tested. In the final system the counters would be placed inside high-pressure thin-walled metal tubes arranged in multiple layers. The test system is now working with a ${ }^{90} \mathrm{Sr}$ source and rise times as low as 1.5 ns have been measured at 20 atmospheres pressure. The slopes get as fast as 30 $\mathrm{ps} / \mathrm{mv}$ for $40 \mathrm{mv}$ raw signals. After the proof of principle is accomplished a 400 cell prototype will be built and then a practical detector designed.

\section{Status of STAR/RLQI\#3 Merger}

The STAR (formerly RLOI\#5) and RLOI\#3 Collaborations have considerable overlap of physics and detector interests. The two collaborations have entered into a joint RHIC R\&D project to determine the performance of Time Projection Chambers (TPC) at RHIC, to optimize the configuration of a TPC-based experiment and to perform TPC tests. Results of this R\&D effort describing the operation and the performance of TPCs at RHIC were presented in the Open Meeting on RHIC Detectors in April at BNL. In addition, the two collaborations have met in April and in June to discuss results and have entered into merger discussions as outlined in the memorandum below. A merger board was formed and has met on two occasions to this date with plans for further meetings.

\section{Memorandum of Understanding - June 20, 1991}

Following discussions between the STAR and RLOI3 collaborations, there is agreement that:

$\rightarrow$ there is considerable overlap in the physics goals of the two collaborations and unanimous agreement that a large acceptance TPC spectrometer is an essential component of the RHIC program;

$\rightarrow$ the two collaborations will work together to develop a new collaboration which will pursue the goal of building a linge acceptance TPC spectrometer at RHIC;

$\rightarrow$ a board will be formed with equal representation from each collaboration which is charged to recommend an experimental layout to the two collaborations by August 9, 1991;

$\rightarrow$ the spokespersons of RLOI3 and STAR will continue to discuss the management structure for the new collaboration, recognizing that agreement has not yet been reached on this important mistter and that agreement must be reached by August 25, 1991 for the new collaboration to present its case to the PAC;

$\rightarrow$ a covering letter describing this process will be submitted with the updated letters of intent from the two collaborations. 


\section{Acknowledgements}

The STAR Collaboration wishes to thank the many individuals who have contributed to this document. We thank S. Chase, H. Crawford, V. Eckardt, F. Goulding, M. Gyulassy, D. Nygren, M. Partlan, M. Shapiro, Y. Shao, J. Simon-Gillo, H. Spieler, L. Teitelbaum, X.N. Wang, R. Welsh and G. Zhang for contributions to the development of the physics and detector concepts of STAR. We thank A. Arthur, W. Edwards and D. Shuman for contributions to this Update. We thank Joy Lofdahl for assistance in the preparation of this manuscript. This work was supported in part by the Director, Office of Energy Research, Division of Nuclear Physics of the Office of High Energy and Nuclear Physics of the U.S. Department of Energy under contract DE-AC03-76SF00098.

\section{Appendices}

\section{A. TPC Electronics Costs}

Detailed cost estimates for the STAR TPC electronics are based upon development of the EOS TPC electronics.

\section{B. Detailed Cost-Engineering Plan}

Inherent in these cost estimates are some assumptions. The most important of these assumptions, is that several key engineers currently involved in SDC and other detector projects at LBL will be available to work on this effort. This is reasonable if notification of funding and the funding capital for engineering the STAR proposal is received in time to adjust the engineering work loads.

Included in this Appendix are the following:

a) A cost estimate for mechanical engineering effort during the 1st half of FY ' 92 towards the STAR proposal.

b) A preliminary estimate of the STAR construction schedule.

c) A preliminary estimate of the STAR installation schedule.

d) A list of questions for RHIC and BNL management, some of which affect the cost and schedules.

Included in the expense column of the cost estimate are some limited travel and licensing fees for CAD design and project management software as well as FEA computing time charges.

It should be noted that the preliminary construction schedule assumes that there is engineering design effort for the 2 nd half of fiscal ' 92 . It is necessary to have some engineering effort continue during this time in order to meet the completion date shown, as well as it being an advantage to maintain continuity.

The intent in developing these very preliminary schedules was to be neither optimistic nor pessimistic in the approach. However, in comparing the results with the RHIC guidelines for completion, it was necessary to accelerate the schedule by 3 to 6 months, thus making it perhaps a bit optimistic. This points out the importance of having the right people available and of having an aggressive design and construction funding profile. 
Appendix A

STAR TPC Electronics Costs

\begin{tabular}{|l|r|r|r|}
\hline Summary & $\begin{array}{r}\text { one time cost } \\
(\mathrm{K} \$)\end{array}$ & $\begin{array}{r}\text { incremental } \\
(\$ / \mathrm{pad})\end{array}$ & $100 \mathrm{~K}$ ch total \\
\cline { 1 - 3 }$(\mathrm{K} \$)$
\end{tabular}

"events/sec

*time buckets/pad

raw pixels/(pad sec)

"pads/tpc

*Engineering ( $k \$ / y e a r)$

* input parameter
Basic system parameters

10

512

5120

150000

\section{Other constants}

120 
Preamp-shaper integrated circuit

\begin{tabular}{|l|r|r|r|}
\hline & one time cost & incremental & $100 \mathrm{~K}$ ch total \\
& $(\mathrm{K} \$)$ & $(\$ /$ pad $)$ & $(\mathrm{K} \$)$ \\
\hline silicon & 50 & 1.83 & 233 \\
packaging & & 0.41 & 41 \\
testing & 50 & 0.14 & 64 \\
\hline Total & 100 & 2.38 & 338 \\
\hline
\end{tabular}

$\begin{array}{lrl}\text { silicon setup cost }(K \$)(\mathrm{a}) & 50 & \text { good chips/tpc } \\ \text { silicon cost/wafer }(\$)(\mathrm{a}) & 2000 & \text { wafers/tpc } \\ \text { pads/die (b) } & 4 & \\ \text { chips/waffer (c) } & 450 & \\ \% \text { yield (d) } & 70 & \\ \% \text { spares } & 15 & \\ \text { package cost/chip (\$) (e) } & 1 & \\ \text { test tech. } \$ / \mathrm{hr} & 20 & \\ \text { test rate chips/hr } & 40 & \\ \text { test fixture }(K \$)(\mathfrak{f}) & 50 & \end{array}$

a) The cost of silicon is based on the EOS preamp order from Orbit.

b) For costing we have used 4 channels per chip since it is not obvious that increasing the channels per die will result in savings. More channels per chip will increase the silicon costs since the yield would be reduced and potential savings in packaging would require the additional setup cost of "on wafer testing".

c) The raw number of chips per wafer is estimated to be $1 / 2$ of the amount obtained in the EOS preamp run since the size of the shaper currently being developed is roughly the same size as the preamp die.

d) Same yield as achieved in the EOS preamp production run.

e) Packaging cost is for plastic by a local manufacturer that is willing to bid on jobs of this size. There is a setup charge which has not been included and will require further investigation.

f) The cost for the testing fixture includes engineering and programming.

Testing costs are based on EOS experience with testing after packaging.

Engineering costs for the integrated circuit have not teen included. It is assumed that this will be covered by RHIC R\&D money. 
SCA-ADC integrated circuit

\begin{tabular}{|l|r|r|r|}
\hline & one time cost & incremental \\
$(\mathrm{K} \$)$ & $100 \mathrm{~K}$ ch total \\
$(\$ / \mathrm{pad})$ & $(\mathrm{K} \$)$ \\
\hline silicon & 50 & 2.88 & 338 \\
packaging & & 0.14 & 14 \\
testing & 50 & 0.07 & 57 \\
\hline Total & 100 & 3.09 & 409 \\
\hline
\end{tabular}

$\begin{array}{lrlr}\text { silicon setup cost }(K \$) \text { (a) } & 50 & \text { good chips/tpc } & 10781 \\ \text { silicon cost/wafer (\$) (a) } & 2000 & \text { wafers/tpc } & 216 \\ \text { channels/die (b) } & 16 & \\ \text { chips/waffer (c) } & 100 & \\ \% \text { yield (d) } & 50 & \\ \% \text { spare } & 15 & \\ \text { package cost/chip (\$) (e) } & 1 & \\ \text { test tech. } \$ / h r & 20 & \\ \text { test rate chips/hr } & 40 & \\ \text { test fixture (K\$) (f) } & 50 & \end{array}$

a) The cost of silicon is based on NA35 SCA Orbit cost (1.2 micron technology).

b) This is what has been done for the NA35 SCA in 1.2 micron technology.

c) Based on EOS SCA with the same dimensions.

d) Same as the EOS SCA yield.

e) Packaging cost is for plastic by a local manufacturer that is willing to bid on jobs of this size. There is a setup charge which has not been included and will require further investigation.

f) The cost for the testing fixture includes engineering and programming.

Testing costs are based on EOS experience with testing after packaging.

Engineering costs for the integrated circuit have not been included. It is assumed that this will be covered by RHIC R\&D money. 
Readout board and data link

\begin{tabular}{|l|r|r|r|}
\hline & $\begin{array}{r}\text { one time cost } \\
(\mathrm{K} \$)\end{array}$ & $\begin{array}{r}\text { incremental } \\
(\$ / \mathrm{pad})\end{array}$ & $100 \mathrm{~K}$ ch total \\
& & 2.63 & 263 \\
\hline hardware without SCA & 240 & & 240 \\
engineering & 240 & 2.63 & 503 \\
\hline total & & & \\
\hline
\end{tabular}

"SCA's/board

3 xilinx (\$)

$\operatorname{taxi}(\$)$

bitbus (\$)

optical transmitter $(\$)$

optical fiber (\$)

optical receiver $(\$)$

taxi receiver (\$)

xilinx receiver $(\$)$

2 pc boards ( $\$$ )

load $2 \mathrm{pc}$ boards $(\$)$

total (\$/read out unit)

$\%$ spares

engineering and testing $(k \$)$
16

90

30

30

20

62

27

24

50

140

140

613

10

240 pads/board

boards/tpc

256

645

data rate (a)

Mbytes/sec

1.31

The total cost of the data link has been included in this device since there is one data link per readout unit. The receiver parts will actually be located on the receiver VME board.

a)This is the data transfer rate this hardware configuration demands. It should be less than $15 \mathrm{Mbytes} / \mathrm{sec}$, the limit for the optical fiber data link.

- input parameter 
Power supplies to detector

\begin{tabular}{|l|r|r|r|}
\hline & $\begin{array}{r}\text { one time cost } \\
(\mathrm{K} \$)\end{array}$ & $\begin{array}{r}\text { rincremental } \\
(\$ / \mathrm{pad})\end{array}$ & $100 \mathrm{~K}$ ch total \\
& 0 & 1.60 & 160 \\
\hline parts and cables & 0 & 0.70 & 70 \\
technicians & 120 & & 120 \\
engineering & 120 & 2.30 & 350 \\
\hline total & & & 1.0 \\
\hline
\end{tabular}

"read out boards/supply

parts and cables (\$/unit)

technician (\$/unit)

engineering $(k \$)$
1

410

180

120
256

586

This system includes power supplies for all the electronics on the detector. There are several voltages to support analog and digital circuits. Power is distributed using one multiconnector cable from each supply unit to each readout card.

Unit costs are scaled from the EOS project which used 128 units. The parts and cables costs include cost of commercial power supplies. The technician time includes cost of assembling control circuitry.

* input parameter 
VME receiver card system

\begin{tabular}{|l|r|r|r|}
\hline & one time cost & incremental & $100 \mathrm{~K}$ ch total \\
& $(\mathrm{K} \$)$ & $(\$ / \mathrm{pad})$ & $(\mathrm{K} \$)$ \\
\hline VME receiver crates & 0 & 1.46 & 146 \\
Receiver VME boards & 0 & 10.74 & 1074 \\
Event builder system & 0 & 0.33 & 33 \\
engineering & 480 & & 480 \\
\hline total & 480 & 12.54 & 1734 \\
\hline
\end{tabular}

"data linkNME board

-DSPs/NME board

-VME boards/crate

*recv crates/event crate

$\%$ spares

VME receiver crate:

crate (\$)

processor (\$)

VSB master (\$)

total

Receiver VME board:

components

xilinx

DSP + memory

PC board, panel etc

total

Event Builder:

crate

processor

memory modules

total

engineering $(K \$)$

\section{2}

16

16

16

10

4000

4000

2500

10500 pads/board

pads/DSP

DSPs/tpc

boards/tpc

crates/tpc

event crts/tpc

DSP (a)

$\mathrm{k}$ pixels/sec
512

32

4688

293

19

2

164

a) This is the processing rate this hardware configuration demands. It should be less than 200k pixels/sec, the maximum expected capability of the EOS TPC DSP.

* input parameter 


\section{Appendix B}

\begin{tabular}{|c|c|c|c|c|c|c|c|}
\hline Subsystem & Engineer & Designer & Admin. & Expense & Engineer & Expense & Total Cost \\
\hline \multicolumn{8}{|c|}{ STAR Engineering Effort Towards Proposal During 1 st \& 2 nd Quarters FY92 } \\
\hline & \multicolumn{3}{|c|}{ Mechanical } & \multicolumn{3}{|c|}{ Electronic } & \multirow{3}{*}{$\frac{\$ K}{\text { Total Cost }}$} \\
\hline & \multicolumn{3}{|c|}{ Effort in Man-Months } & $\$ K$ & Effort & SK & \\
\hline Subsystem & Engineer & Designer & Admin. & Expense & Engineer & Expense & \\
\hline & & & & & & & \\
\hline 1) Silicon Drift Chamber & & & & & & & $\$ 197.33$ \\
\hline Requirements & 1 & & & & 2 & & \\
\hline Design Concept & 1 & 3 & & & 6 & $\$ 2.00$ & \\
\hline Analysis & 2.75 & & & $\$ 2.75$ & 1 & & \\
\hline Costing & 1 & & & & 2 & & \\
\hline & & & & & & & \\
\hline 2) Hi-eta Detectors & & & & & & & $\$ 78.28$ \\
\hline Requirements & 0.25 & & & & 1 & & \\
\hline Design Concept & 0.5 & 1 & & & 2 & $\$ 2.00$ & \\
\hline Analysis & 0.5 & & & $\$ 0.50$ & 1 & & \\
\hline Costing & 0.5 & & & & 1 & & \\
\hline & & & & & & & \\
\hline 3) Time of Flight Start & & & & & & & $\$ 48.40$ \\
\hline Requirements & 0.5 & & & & 0.25 & & \\
\hline Design Concept & 0.5 & 1 & & & 0.5 & $\$ 2.00$ & \\
\hline Analysis & 0.5 & & & $\$ 0.50$ & 0.5 & & \\
\hline Costing & 0.5 & & & & 0.5 & & \\
\hline & & & & & 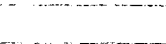 & & \\
\hline 4) TPC & & & & & & & $\$ 189.70$ \\
\hline Requirements & 1 & & & & 2 & & \\
\hline Design Concept & 3 & 3 & & & 4 & $\$ 2.00$ & \\
\hline Analysis & 2 & & & $\$ 2.00$ & 1 & & \\
\hline Costing & 1 & & & & 2 & & \\
\hline & & & & & & & \\
\hline 5) Time of Flight & & & & & & & $\$ 94.80$ \\
\hline Requirements & 1 & & & & 0.5 & & \\
\hline Design Concept & 1 & 2 & & & 1 & $\$ 2.00$ & \\
\hline Analysis & 1 & & & $\$ 1.00$ & 1 & & \\
\hline Costing & 1 & & & & 1 & & \\
\hline & & & & & 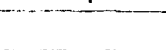 & & \\
\hline 6) Solenoid & & & & & & & $\$ 47.30$ \\
\hline Requirements & 0.5 & & & & & & \\
\hline Design Concept & 1 & 2 & & & & & \\
\hline Analysis & 1 & & & $\$ 1.00$ & & & \\
\hline Costing & 0.5 & & & & & & \\
\hline & & & & & & & \\
\hline 7) Yoke/Supports & & & & & & & $\$ 97.70$ \\
\hline Requirements & 2 & & & & & & \\
\hline Design Concept & 2 & 3 & & & & & \\
\hline Analysis & 2 & & & $\$ 2.00$ & & & \\
\hline Costing & 1 & & & & & & \\
\hline & & & & & & & \\
\hline 8) EM Calorimeter & & & & & & & $\$ 101.35$ \\
\hline Requirements & 1 & & & & 1 & & \\
\hline Design Concept & 1.5 & 1.5 & & & 1 & $\$ 2.00$ & \\
\hline Analysis & 1 & & & $\$ 1.00$ & 1 & & \\
\hline Costing & 1 & & & & 1 & & \\
\hline & & & & & & & \\
\hline
\end{tabular}




\begin{tabular}{|c|c|c|c|c|c|c|c|}
\hline Subsystem & Engineer & Designer & Admin. & Expense & Engineer & Expense & Total Cost \\
\hline \multicolumn{2}{|c|}{ 9) Computing (Online, Offline) } & & & & & & $\$ 0.00$ \\
\hline \multicolumn{2}{|c|}{ Requirements } & & & & & & \\
\hline Design Concept & & \multicolumn{2}{|c|}{ (no estimate) } & & & & \\
\hline \multicolumn{8}{|l|}{ Analysis } \\
\hline \multicolumn{8}{|l|}{ Costing } \\
\hline & & & & & & & \\
\hline 10) Utilities & & & & & & & $\$ 38.90$ \\
\hline Requirements & 1 & & & & & & \\
\hline Design Concept & 1 & 1 & & & & & \\
\hline \multicolumn{8}{|l|}{ Analysis } \\
\hline \multirow[t]{2}{*}{ Costing } & 1 & & & & & & \\
\hline & & & & & & & \\
\hline 11) Installation \& Test & & & & & & & $\$ 48.13$ \\
\hline Requirements & 0.25 & & & & 0.25 & & \\
\hline Design Concept & 1 & & & & 1 & & \\
\hline \multicolumn{8}{|l|}{ Analysis } \\
\hline \multirow[t]{2}{*}{ Costing } & 1 & & & & 1 & $\$ 2.00$ & \\
\hline & & & & & & & \\
\hline \multicolumn{2}{|l|}{ 12) Project Management } & & & & & & $\$ 77.50$ \\
\hline Requirements & 1 & & & & & & \\
\hline Design Concept & 2 & & & & & & \\
\hline \multicolumn{8}{|l|}{ Analysis } \\
\hline Costing & 3 & & 3 & $\$ 1.00$ & & & \\
\hline \multirow[t]{2}{*}{ Other } & & & 3 & $\$ 4.00$ & & & \\
\hline & & & & & & & \\
\hline \multicolumn{2}{|c|}{ 13) Integration Management } & & & & & & $\$ 97.60$ \\
\hline Requirements & 1 & & & & & & \\
\hline Design Concept & 4 & 4 & & $\$ 5.00$ & & & \\
\hline \multicolumn{8}{|l|}{ Analysis } \\
\hline Costing & 1 & & & & & & \\
\hline \multirow[t]{2}{*}{ Other } & & & & $\$ 4.00$ & & & \\
\hline & & & & & & & \\
\hline \multirow[t]{2}{*}{ Total (Man-Months/\$K) : } & 52.25 & 21.5 & 6 & $\$ 24.75$ & 36.5 & $\$ 14.00$ & $\$ 1,116.98$ \\
\hline & & & & & & & $(\$ 1,731.31)$ \\
\hline \multirow{2}{*}{\multicolumn{8}{|c|}{$\begin{array}{l}\text { Note: Labor rates per man-month assumed to be LBL - WITHOUT OVERHEAD; } \\
\text { ie, Equipment } \$ \text { (with Overhead is in ()) }\end{array}$}} \\
\hline & & & \\
\hline 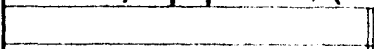 & \$ K $/ \mathrm{mo}$ & 2 & & & & & \\
\hline Elec. Engineer & $\$ 10.00$ & & & & & & \\
\hline Moch. Engineer & $\$ 10.50$ & & & & & & \\
\hline Designer & $\$ 7.40$ & & & & & & \\
\hline \multirow[t]{2}{*}{ Administrator } & $\$ 4.50$ & & & & & & \\
\hline & & & & & & & \\
\hline \multicolumn{7}{|c|}{ Note: There is no estimate included here for Computing effort towards the proposal. } & \\
\hline & & & & & & AAA $7 / 12 / 9$ & WRE $7 / 5 / 91$ \\
\hline
\end{tabular}




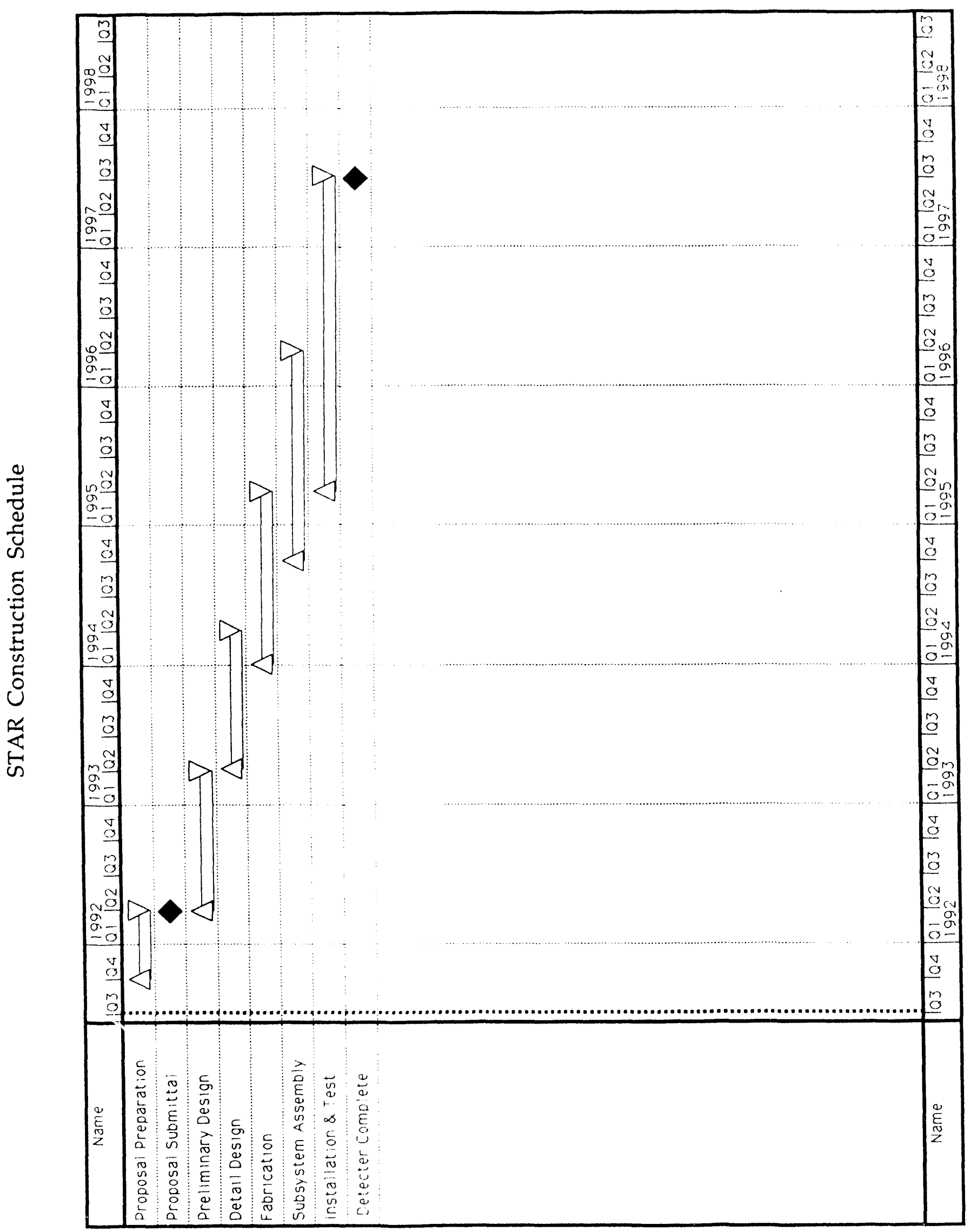




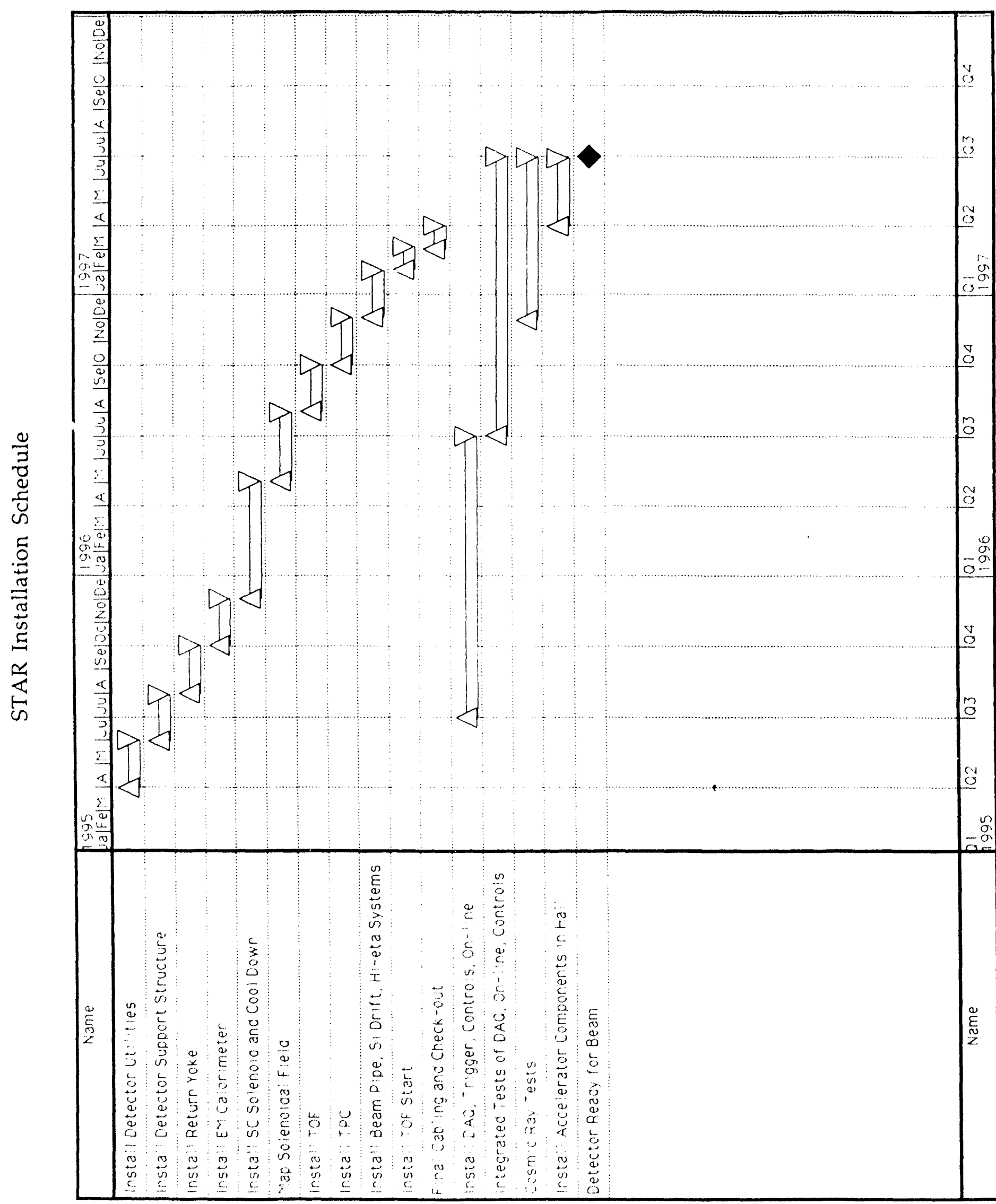




\section{Questions about the engineering estimate:}

1) In FY '92 will the money be equipment or operating?

2) What level of detail is expected for the proposal:
a) for the Design?
b) for the Cost Estimate?

3) Project Management System Requirements? (ie., how often do we report progress, costs, effort remaining, etc.)

4) Which subsystems will be handled by which institutions?

5) How will computing be handled, and by whom?

6) Is BNL (or will they at sometime be) interested in taking over Project Management and Integration Management?

7) What is the real construction schedule?

8) What is source of funding, how serious and how far along is it?

9) What is the likelihood of second half FY92 funding?

10) What is the availability of key physicists during Sept., Oct., Nov '91 time frame? (to get a jump on su'ssystem requirements) 

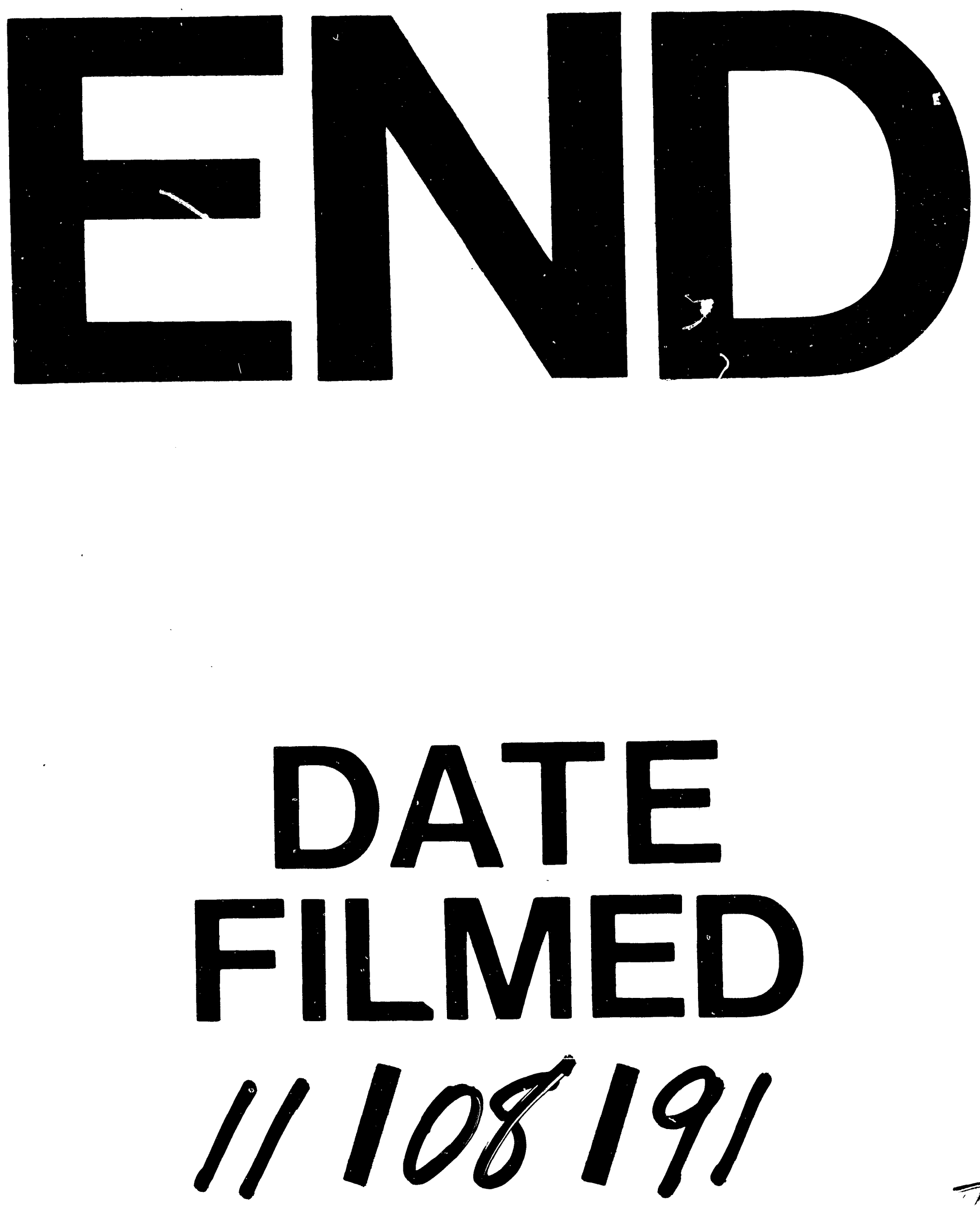

I 
